
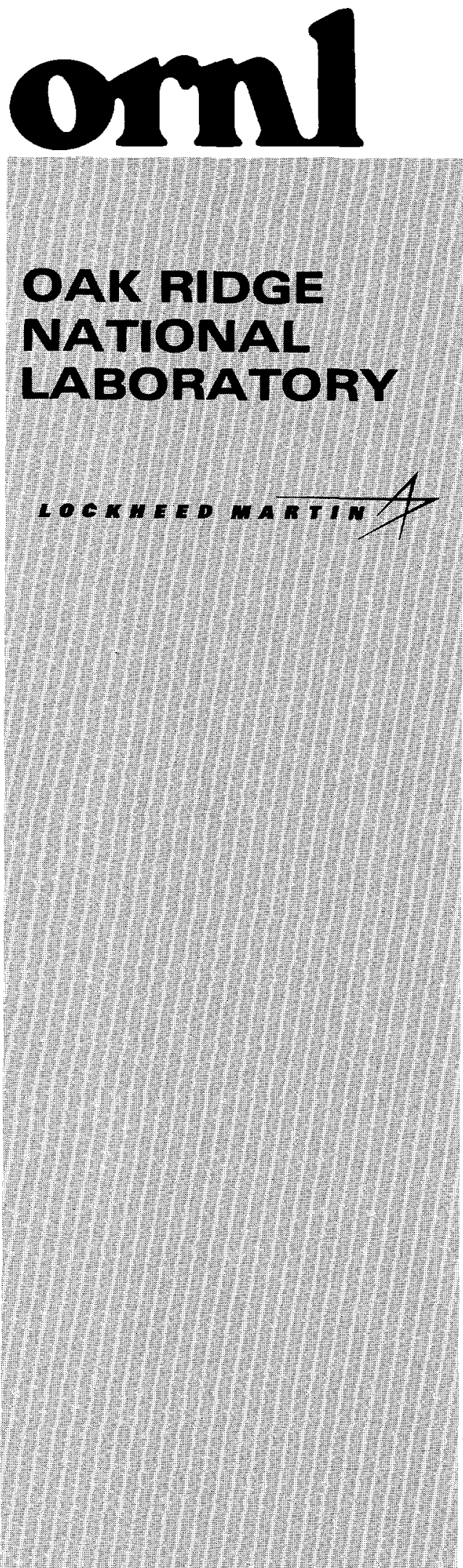

WAMGED ANO OPERATEO BY

LOCKHEEO WATIN EHERGY RESEARCH CORPORATION FOR THE UNTED STATES

DEPARTIUENT OF ENERG:

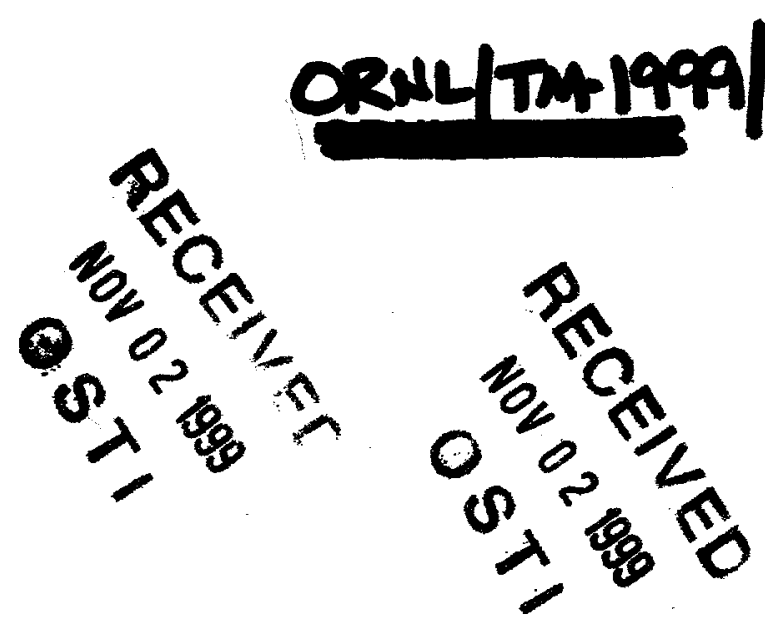

Application of Global Optimization to the Estimation of Surface-Consistent Residual Statics

D. B. Reister, Edward M. Oblow, Jacob Barhen, and J. B. DuBose, Jr. 
This report has been reproduced directly from the best available copy.

Available to DOE and DOE contractors from the Office of Scientific and Technical Information, P. O. Box 62, Oak Ridge, TN 37831; prices available from (423) 576-8401.

Available to the public from the National Technical Information Service, U.S. Department of Commerce, 5285 Port Royal Rd., Springfield, VA 22161.

This report was prepared as an account of work sponsored by an agency of the United States Government. Neither the United States nor any agency thereof, nor any of their employees, makes any warranty, express or implied, or assumes any legal liability or responsibility for the accuracy, completeness, or usefulness of any information, apparatus, product, or process disclosed, or represents tat its use would not infringe privately owned rights. Reference herein to any specific commercial produce, process, or service by trade name, trademark, manufacture, or otherwise, does not necessarily constitute or imply its endorsement, recommendation, or favoring by the United States Government or any agency thereof. The views and opinions of authors express herein do not necessarily stat or reflect those of the United States Government or any agency thereof. 


\section{DISCLAIMER}

Portions of this document may be illegible in electronic image products. Images are produced from the best available original document. 


\title{
APPLICATION OF GLOBAL OPTIMIZATION TO THE ESTIMATION OF SURFACE-CONSISTENT RESIDUAL STATICS
}

\author{
${ }^{\dagger}$ D. B. Reister \\ ${ }^{\dagger}$ E. M. Oblow \\ ${ }^{\dagger} \mathrm{J}$. Barhen

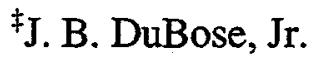 \\ ${ }^{\dagger}$ Center for Engineering Science Advanced Research \\ Computer Science and Mathematics Division \\ Oak Ridge National Laboratory \\ P. O. Box 2008-6355 \\ Oak Ridge, TN 37831 \\ ${ }^{\ddagger}$ Paradigm Geophysics \\ 1200 Smith St., Suite 2100 \\ Houston, TX 77002
}

October 1999
Research sponsored by the Office of Fossil Energy
(Office of Natural Gas and Petroleum Technology) and the Office of Science
(Office of Advanced Scientific Computing Research and Office of Basic Energy Sciences) of the U. S. Department of Energy (DOE)

\author{
Prepared by \\ OAK RIDGE NATIONAL LABORATORY \\ Oak Ridge, Tennessee 37831-6285 \\ managed by \\ LOCKHEED MARTIN ENERGY RESEARCH CORP. \\ for the \\ U.S. DEPARTMENT OF ENERGY \\ under Contract DE-AC05-96OR22464
}


CONTENTS

Page

LIST OF FIGURES

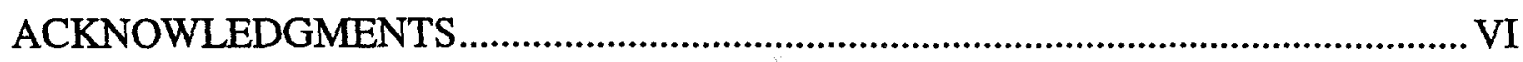

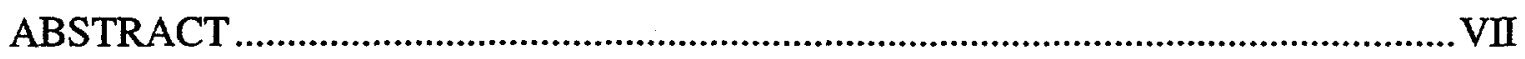

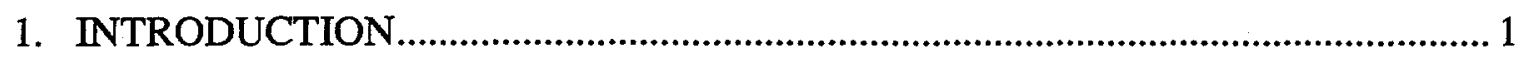

2. THE RESIDUAL STATICS OPTIMIZATION PROBLEM.......................................

2.1 STACK POWER

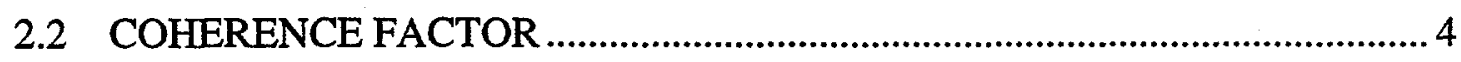

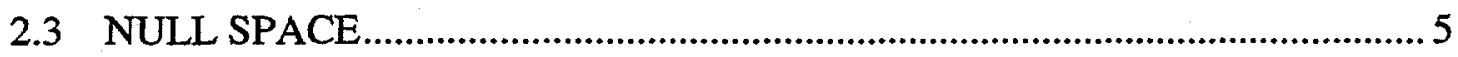

2.4 DECOUPLE THE COMPONENTS OF THE STACK POWER …..................... 7

2.5 NULL SPACE FOR THE DECOUPLED PROBLEMS ………….......................

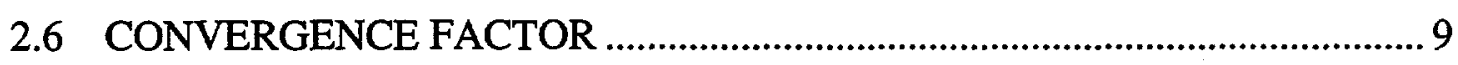

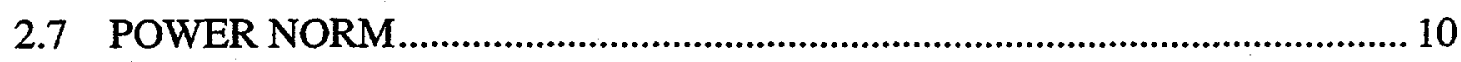

3. GLOBAL OPTIMIZATION ALGORITHM .....................................................

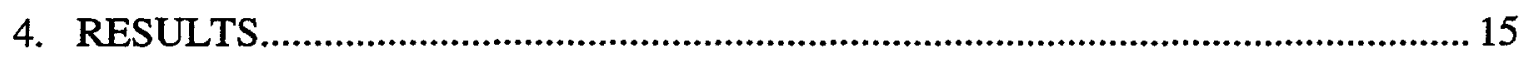

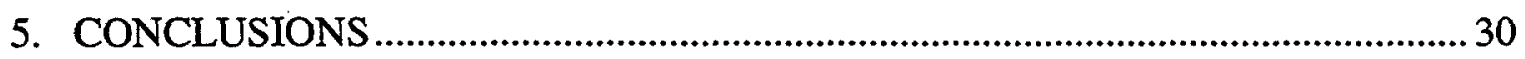

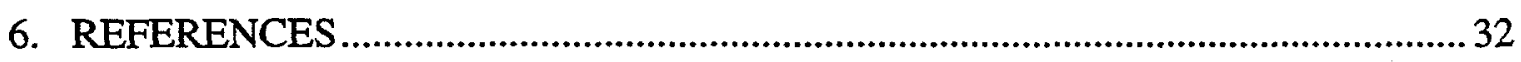





\section{LIST OF FIGURES}

\section{Figures}

Page

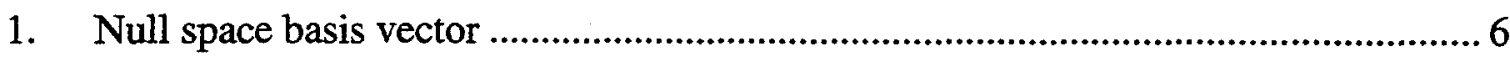

2. Row 52 of the $B$ matrix for the example problem ................................................ 9

3. The mapping between the decoupled problems and the large problem.................... 10

4. Finding the global minimum for a 1D example using the SPT algorithm................ 14

5. The seismic image before the application of the disrupting statics ......................... 17

6. The disrupted seismic image before the application of residual statics corrections 18

7. The seismic image after the application of residual statics corrections.................... 19

8. The stack power $(\mathrm{E})$ and the upper bound $(\mathrm{G})$ for each DMP ............................... 20

9. The best coherence factor ................................................................................. 20

10. The difference between the current value of the stack power and the best value for

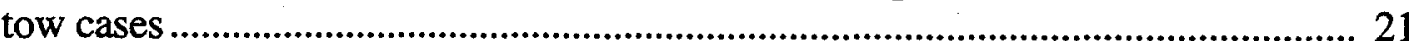

11. The convergence factor for two cases............................................................... 22

12. The Euclidean distance between the 22 vectors after null space corrections ........... 22

13. The null space correction for the distances between 22 vectors.............................. 23

14. The power norm distance between the 22 vectors ................................................. 23

15. Correlation between the power norm and Euclidean distance................................. 24

16. Euclidean distance versus the stack power ......................................................... 25

17. Power norm versus the stack power ................................................................. 25

18. Power norm versus the power difference ……................................................... 26

19. The difference between the power norm and the power differences versus the stack power

20. The disrupting statics that were applied to the original seismic data ....................... 28

21. The difference between the statics for the 2441 case and the disrupting statics ..... 28

22. The difference between the statics for the 2427 case and the disrupting statics .... 29

23. The difference between the statics for the 2441 case and the 2427 case................ 29

24. The difference between the statics for the 2415 case and the disrupting statics ..... 30 



\section{Acknowledgments}

This work was sponsored by both the Office of Fossil Energy (Office of Natural Gas and Petroleum Technology) and the Office of Science (Office of Advanced Scientific Computing Research and Office of Basic Energy Sciences), U.S. Department of Energy (DOE). Oak Ridge National Laboratory is managed for DOE by Lockheed Martin Energy Research Corporation under contract number DE-AC05-96OR22464. 


\begin{abstract}
Since the objective function that is used to estimate surface-consistent residual statics can have many local maxima, a global optimization method is required to find the optimum values for the residual statics. As reported in several recent papers, we had developed a new method (TRUST) for solving global optimization problems and had demonstrated it was superior to all competing methods for a standard set of nonconvex benchmark problems. The residual statics problem can be very large with hundreds or thousands of parameters, and large global optimization problems are much harder to solve than small problems. To solve the very challenging residual statics problem, we have made major improvements to TRUST (Stochastic Pijavskij Tunneling) and we have made several significant advances in the mathematical description of the residual statics problem (derivation of two novel stack power bounds and disaggregation of the original problem into a large number of small problems). Using the enhanced version of TRUST, we have performed extensive simulations on a realistic sample problem that had been artificially created by large static disruptions. Our simulations have demonstrated that TRUST can reach many plausible distinct "solutions" that could not be discovered by more conventional approaches. An unexpected result was that high values of the stack power may not eliminate cycle skips.
\end{abstract}




\section{INTRODUCTION}

Since the earliest days of seismic exploration, geophysicists have recognized the need to correct for the low velocity in the weathered and unconsolidated sediments near the earth's surface. The data processing procedure has been described by Yilmaz (1987), Marsden [1993], and Sheriff and Geldart [1995]. The first corrections for elevation and low velocity are field statics. A reference level is determined that is below the low velocity layer (LVL), and field statics move the sources and receivers to the reference level. Common midpoint (CMP) gathers are used to generate a set of preliminary velocity picks that are used to calculate normal move out (NMO) corrections. Residual statics corrections are calculated using the corrected data. The process is repeated (velocity picks, NMO corrections, and residual statics) until the results converge.

The conventional method for calculating residual statics corrections was developed in the seminal papers of Taner et al. [1974] and Wiggins et al. [1976]. The time delays caused by the passage of seismic signals through the LVL should depend on path. After the NMO corrections, we assume that all of the paths are vertical and estimate a single time delay that is "surface consistent" (each source and receiver location has a time delay that does not depend on the wave path). The time delay $\left(T_{\text {srk }}\right)$ for a trace that follows a path from a source (s) to a receiver (r) via a common midpoint $(\mathrm{k})$ is determined by maximizing the cross-correlation between the trace and the CMP gather. The total time delay has four components: the source static $\left(\mathrm{S}_{\mathrm{s}}\right)$, the receiver static $\left(\mathrm{R}_{\mathrm{r}}\right)$, the two-way travel time from the reference level to a reference subsurface reflector $\left(\Gamma_{\mathrm{k}}\right)$, and a residual NMO correction $\left[\mathrm{M}_{\mathrm{k}}\left(\mathrm{X}_{\mathrm{sr}}\right)^{2}\right]$ where $\mathrm{X}_{\mathrm{sr}}$ is the distance from the source to the receiver [see Eq. (1)].

$$
\mathrm{T}_{\mathrm{srk}}=\mathrm{S}_{\mathrm{s}}+\mathrm{R}_{\mathrm{r}}+\Gamma_{\mathrm{k}}+\mathrm{M}_{\mathrm{k}} \mathrm{X}_{\mathrm{sr}}^{2}
$$

Although the total time delay $\left(\mathrm{T}_{\text {srk }}\right)$ is an independent value for each trace, the parameters on the right side of Eq. (1) each occur in many traces. Thus, the parameters are over determined (we have many more equations than parameters). We will consider a problem with 4776 traces, 100 shots, 216 receivers, and 423 common midpoints. For our problem we have 4776 equations and 1162 parameters. Since the equations are over determined, they can only be solved by minimizing the errors (by using the method of least squares).

Taner discovered that the problem is both over determined and underdetermined. Although there are many more equations than unknowns, he found sets of nonzero parameters that satisfy Eq. (1) when all of the total time delays are zero. These solutions form a null space and we can add an arbitrary linear combination of the vectors in the null space to any solution to Eq. (1) and still have a solution. Taner added an extra set of terms to the least squares objective function to eliminate the null space solutions.

Wiggins clarified the work of Taner by applying singular value decomposition to find the least squares solution of Eq. (1), by displaying the eigenvectors for a small problem, and by introducing the term "null space." 
One of the conventional methods for calculating residual statics corrections has two steps: use cross correlation to estimate the total time delay $\left(\mathrm{T}_{\text {srk }}\right)$ for each trace and use least squares to find the parameters in Eq. (1). In 1985, Ronen and Claerbout proposed a onestep alternative approach: stack power maximization. They defined an objective function that measures the correlation between all of the traces in each CMP gather. Changes in the parameters in Eq. (1) cause a time shift for each trace and change the correlation between traces. They search for parameter values that maximize the stack power.

If synthetic data is created by time shifting a trace, correlation of the two traces will identify the time shift (the correlation will be 1.0 at the proper time shift). For real data, there may be many local maxima in the correlation function. The failure to find the largest local maxima results in a "cycle skip." The stack power function depends on thousands of traces and hundreds of parameters and can have a very large number of local maxima. If there are $\mathrm{N}$ parameters and $\mathrm{M}$ local maxima in each dimension of the $\mathrm{N}$ dimensional space, the logarithm of the total number of local maxima is $N \log M$. Most optimization methods will find a local maximum. A problem with many local maxima requires a global optimization method.

In 1985, Rothman recognized that the residual statics problem was a global optimization problem and proposed to solve the problem using the simulated annealing method. $\mathrm{He}$ defined the stack power optimization using two of the four types of parameters on the right side of Eq. (1); his parameters are the static corrections for the sources and receivers. Since the total stack power is a sum of the stack power for each CMP, the CMP term $\left(\Gamma_{\mathrm{k}}\right)$ shifts all of the traces in the stack and does not change the stack power. He argued that the residual NMO correction $\left[\mathrm{M}_{\mathrm{k}}\left(\mathrm{X}_{\mathrm{sr}}\right)^{2}\right]$ was cumbersome to estimate and had a small impact on the stack power. In 1986, Rothman improved his method and applied it to some field data that required large static corrections (up to $200 \mathrm{~ms}$ ).

In 1993, DuBose proposed several innovations to improve Rothman's method. The simulated annealing method depends on a parameter: the "temperature." DuBose proposed an algorithm for finding the proper temperature. To apply his method, he measures the changes in the statics from one iteration to the next. If the changes are too small, he raises the temperature. If the changes are too large, he reduces the temperature. Since he performs calculations in the frequency domain, each trace can be stored compactly and time shifting is performed by an efficient multiplication by a complex number. In the time domain, each trace is sampled at a regular period and crosscorrelation for a time shift that is a fraction of a sampling step requires interpolation. No interpolation is required in the frequency domain.

DuBose assumes that receiver statics are constrained with position along the line such that the change from one receiver to the next is bounded by a value substantially smaller than the largest possible static. He also assumes that any shot that is very close to a receiver station should have its static constrained within another modest limit to be near the static of its receiver station. These assumptions limit the regions in parameter space 
that need to be searched. He modifies the objective function by performing a running average over a few adjacent stacked traces. To eliminate the null space from the problem, he adds a penalty term to the objective function.

Other significant contributions to the literature on residual statics include: Marcoux (1981), Taner (1981), Morley (1983), Caklovic (1985), Levy (1987), Levin (1989), Normark (1991), Cambois (1992), Cambois (1993), Normark (1993), and Wilson (1994).

Recently, an improved algorithm for solving global optimization problems was developed at the Center for Engineering Science Advanced Research (CESAR) at the Oak Ridge National Laboratory (ORNL) [see Barhen (1997)]. The algorithm is called TRUST (terminal repeller unconstrained subenergy tunneling). In 1996, we began a collaborative project to apply TRUST to the residual statics problem using an objective function defined by DuBose [the function is not identical to the function used in DuBose (1993)]. The results reported in Barhen (1997) are for a statics problem with fewer parameters and fewer traces than the problem considered here. In applying TRUST to the statics problem, we have made significant improvements in TRUST. Details of the most recent improvements are in Oblow (1999) and will be summarized here.

The next section will define the stack power, derive an upper bound for the stack power, discuss the null space solutions, and define a decoupled stack power. The solution of the decoupled problem both provides an initial estimate of the parameters for the coupled problem and provides a closer upper bound for the stack power of the coupled problem. The third section will discuss both TRUST and the recent improvements in TRUST. The fourth section will discuss our results for a test problem based on field data. The fifth section will present our conclusions.

\section{THE RESIDUAL STATICS OPTIMIZATION PROBLEM}

\subsection{STACK POWER}

Seismic energy is detected by receivers that are located along a line. The source of seismic energy is moved along the line to produce each new shot. Time series data is collected from the receivers for each shot and the source of seismic energy is moved to the next source location for the next shot. The time series data is stored as Fourier coefficients. Common midpoint (CMP) stacking is used to increase the signal-to-noise ratio. The fold of the data is the number of data sets with the same CMP. Between 1960 and 1980, improvements in data acquisition systems allowed typical values for the fold to increase from one to twelve. Today, thirty is a typical value.

Data are provided by trace $\left[t=1, N_{t}\right]$. For each trace, the data consist of the Fourier components $\left[f=1, N_{f}\right]$ of the measured signal. The Fourier components $\left[D_{f t}\right]$ are complex numbers. The seismic energy travels from a source $\left[s_{t}\right]$ to a receiver $\left[r_{t}\right]$ via a midpoint $\left[\mathrm{k}_{\mathrm{t}}\right]$. For each midpoint $(\mathrm{k})$, the data are stacked: 


$$
H_{\mathrm{tf}}=\sum_{\mathrm{t}} \exp \left[2 \pi \mathrm{if}\left(\mathrm{S}_{\mathrm{s}}+\mathrm{R}_{\mathrm{r}}\right)\right] \mathrm{D}_{\mathrm{ft}}
$$

The statics corrections $\left(S_{S}\right.$ and $\left.R_{r}\right)$ are determined to maximize the total power $(E)$ in the stacked data:

$$
\mathrm{E}=\sum_{\mathrm{k}} \sum_{\mathrm{f}}\left|\mathrm{H}_{\mathrm{kf}}\right|^{2}
$$

\subsection{Coherence Factor}

In this subsection, we will develop a metric (that we call a "coherence factor") to measure progress toward the goal of maximizing $\mathrm{E}$. We begin by defining the power for each $\operatorname{CMP}\left(\mathrm{E}_{\mathrm{k}}\right)$ :

$$
\mathrm{E}_{\mathrm{k}}=\sum_{\mathrm{f}}\left|\mathrm{H}_{\mathrm{kf}}\right|^{2}
$$

Then the total power is the sum of the $\mathrm{E}_{\mathrm{k}}$. In this subsection, we will derive an upper bound $\left(G_{k}\right)$ for each of the $E_{k}$. The coherence factor $\left(Q_{k}\right)$ is the ratio of $E_{k}$ and $G_{k}$ :

$$
\mathrm{Q}_{\mathrm{k}}=\mathrm{E}_{\mathrm{k}} / \mathrm{G}_{\mathrm{k}}
$$

Since the $E_{k}$ are positive, each of the coherence factors will be in the range $[0,1]$.

To begin our derivation of the upper bound $\left(G_{k}\right)$, the complex numbers $\left[D_{f t}\right]$ will be written in polar form:

$$
D_{\mathrm{ft}}=\alpha_{\mathrm{ft}} \exp \left(\mathrm{i} \theta_{\mathrm{ft}}\right)
$$

Using the polar form of the data, Eq. (2) becomes:

$$
H_{\mathrm{lf}}=\sum_{\mathrm{t}} \exp \left[2 \pi \mathrm{if}\left(\mathrm{S}_{\mathrm{s}}+\mathrm{R}_{\mathrm{r}}\right)\right] \alpha_{\mathrm{ft}} \exp \left(\mathrm{i} \theta_{\mathrm{ft}}\right)
$$

We define $\psi_{\mathrm{ft}}$ by:

$$
\Psi_{\mathrm{ft}}=2 \pi f\left(\mathrm{~S}_{\mathrm{s}}+\mathrm{R}_{\mathrm{r}}\right)+\theta_{\mathrm{ft}}
$$

Using $\psi_{\mathrm{ft}}$, Eq. (7) may be written: 


$$
H_{k f}=\sum_{t} \alpha_{f t} \exp \left(i \psi_{f t}\right)
$$

Using our expression for $\mathrm{H}_{k f}$, we can derive a new expression for $\mathrm{E}_{\mathrm{k}}$ :

$$
E_{k}=\sum_{f} \sum_{t} \alpha_{f t} \exp \left(i \psi_{f t}\right) \sum_{\tau} \alpha_{f_{\tau}} \exp \left(-i \psi_{f_{\tau}}\right)
$$

Regrouping terms:

$$
E_{k}=\sum_{f} \sum_{t} \sum_{\tau} \alpha_{f t} \alpha_{f \tau} \exp \left[i\left(\psi_{f t}-\psi_{f \tau}\right)\right]
$$

If we add Eq. (11) to the same equation with the indexes $t$ and $\tau$ reversed:

$$
2 E_{k}=\sum_{f} \sum_{t} \sum_{\tau} \alpha_{f t} \alpha_{f \tau}\left[\exp \left[i\left(\psi_{f t}-\psi_{f \tau}\right)\right]+\exp \left[-i\left(\psi_{f t}-\psi_{f \tau}\right)\right]\right]
$$

Using the definition of $\cos \mathrm{z}$, Eq. (12) may be simplified:

$$
E_{k}=\sum_{f} \sum_{\tau} \sum_{\tau} \alpha_{f t} \alpha_{f_{\tau}} \cos \left(\psi_{f t}-\psi_{f \tau}\right)
$$

We define the upper bound for $E_{k}\left(G_{k}\right)$ by:

$$
G_{k}=\sum_{f} \sum_{t} \sum_{\tau} \alpha_{f t} \alpha_{f \tau}=\sum_{f}\left(\sum_{t} \alpha_{f t}\right)^{2}
$$

Since the upper bound for the $\cos \mathrm{z}$ is one, $\mathrm{G}_{\mathrm{k}}$ is an upper bound for $\mathrm{E}_{\mathrm{k}}$.

\subsection{Null Space}

In his 1974 paper, Taner derived analytical expressions for the null space basis vectors for Eq. (1). He found a five-dimensional null space. Since we are not considering the residual NMO correction, Taner's analytical expressions identify a three-dimensional null space for the stack power [Eq. (3)]. The three null space vectors are: (1) a constant for all sources and zero for receivers, (2) a constant for all receivers and zero for sources, and (3) a vector that is proportional to the source position for the sources $\left({ }^{s} X_{s}\right)$ and proportional to the receiver position for the receivers $\left({ }^{\mathrm{R}} \mathrm{X}_{\mathrm{r}}\right)$. To any vector of statics corrections, we can add the null space vectors and not change the total stack power: 


$$
\begin{aligned}
& S_{s} \rightarrow S_{s}+c^{s}+c^{S R}{ }^{s} X_{s} \\
& R_{r} \rightarrow R_{r}+c^{R}+c^{S R ~ R} X_{r}
\end{aligned}
$$

When the expressions on the right sides of Eqs. (15) and (16) are substituted into the expression for the stack [Eq. (2)], the phase of every trace in CMP stack $k$ is shifted by the same constant $\left[\mathrm{X}_{s}+{ }^{R} \mathrm{X}_{\mathrm{r}}\right.$ is a constant for each CMP]. Consequently, there is no change in the stack power. The third null space vector (that depends on the positions of the sources and receivers) is displayed in Fig. 1 for the problem that we shall consider later in this paper.

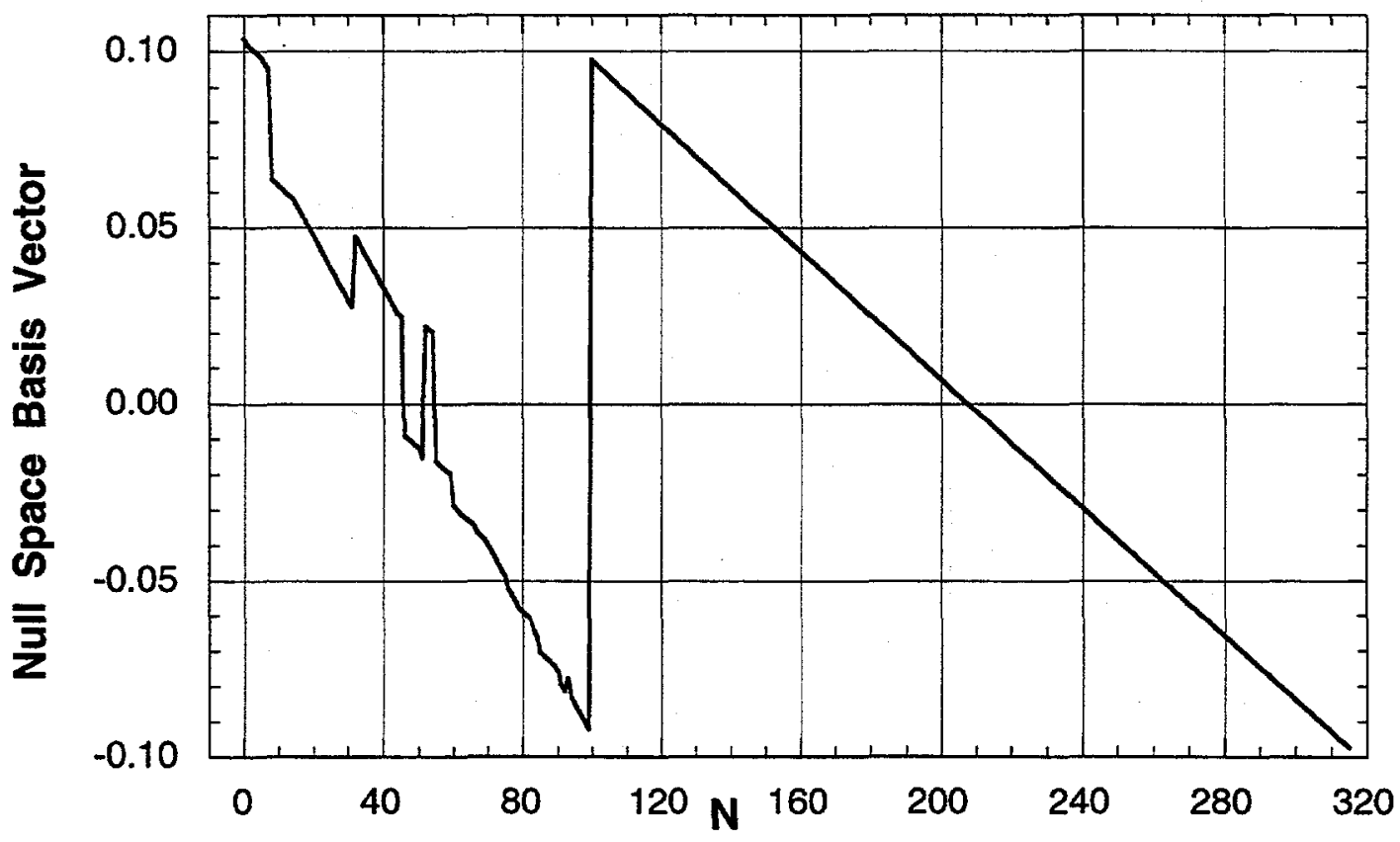

Fig. 1. The third null space vector for a problem with 100 sources and 216 receivers.

Taner added a quadratic term to eliminate the null space components from the statics corrections vector. We prefer not to eliminate the null space components. Given two vectors, we want to know if they are distinct. To compare the vectors, we will find the difference between the vectors, subtract the three null space components and use the Euclidean norm on the residual difference to define the distance between the two vectors.

\subsection{DECOUPLE THE COMPONENTS OF THE STACK POWER}

In Subsection 2.2, we defined the power $\left(\mathrm{E}_{\mathrm{k}}\right)$ and a coherence factor $\left(\mathrm{Q}_{\mathrm{k}}\right)$ for each CMP. However, each of the statics can influence many of the CMP gathers. In this subsection, we introduce new variables that decouple the CMP gathers. The advantage of this approach is that we can solve a large number of small problems rather than one large problem and derive a closer upper bound for the stack power of the coupled problem. 
The solutions of the decoupled problems provide an excellent initial estimate for the parameters for the coupled problem.

For each trace $(t)$, we define $v_{t}$ by:

$$
v_{t}=S_{s_{t}}+R_{r_{t}}
$$

Using matrix notation, Eq. (17) may be written:

$$
\mathbf{v}=\mathbf{A x}
$$

where $\mathbf{x}$ is the parameter vector that combines the vector of source statics corrections (S) and the vector of receiver statics corrections $(\mathbf{R})$ :

$$
\mathbf{x}=\left[\begin{array}{l}
\mathbf{S} \\
\mathbf{R}
\end{array}\right]
$$

Using the new variables, Eq. (2) becomes:

$$
H_{k f}=\sum_{t} \exp \left[2 \pi i f v_{t}\right] D_{f t}
$$

Thus, each of the $\mathrm{H}_{\mathrm{kf}}$ depends on a unique subset of the $\mathrm{v}_{\mathrm{t}}\left[\mathrm{k}_{\mathrm{v}}\right]$ and the problem is decoupled. Using the new variables [ $\left[\mathrm{k}_{\mathrm{v}}\right]$, the components of the stack power are:

$$
E_{k}\left({ }^{k} v\right)=\sum_{f}\left|H_{k f}\right|^{2}
$$

Each of the $\mathrm{K}$ decoupled global optimization problems can be solved independently using TRUST to find the components of the vector $\mathbf{v}\left[\mathrm{v}_{\mathrm{t}}\right]$. We shall call the maximum value for each component of the stack power for the decoupled problem: ${ }^{D} G_{k}$. The optimal coordinates $[v]$ can be mapped back to the original coordinates:

$$
\mathbf{x}=\mathbf{B v}
$$

Using singular value decomposition, the matrix $\mathbf{A}$ can be decomposed into three matrices:

$$
\mathbf{A}=\mathbf{U} \mathbf{W} \mathbf{V}^{\mathrm{T}}
$$

where $\mathbf{U}$ and $\mathbf{V}$ are orthogonal, $\mathbf{W}$ is diagonal with positive elements, and $\mathbf{V}$ is square: $\mathbf{U}^{\mathrm{T}} \mathbf{U}=\mathbf{I}$ and $\mathbf{V} \mathbf{V}^{\mathrm{T}}=\mathbf{V}^{\mathrm{T}} \mathbf{V}=\mathbf{I}$. Using the three matrices, the inverse matrix $\mathbf{B}$ is given by: 


$$
\mathbf{B}=\mathbf{V} \mathbf{W}^{-1} \mathbf{U}^{\mathrm{T}}
$$

The values for one row of the $\mathbf{B}$ matrix for the example that we shall consider in Section 4 are displayed in Fig. 2. The most striking feature of the figure is that only a few values are large and they are all approximately equal. Furthermore, the first large value occurs for trace 2329 and the first trace in the A matrix that has a component in column 52 is trace 2329. We will now demonstrate why the $\mathbf{B}$ matrix has these features and derive an approximate value for the large values of the $\mathbf{B}$ matrix. If we use Eq. (18) to eliminate $\mathbf{v}$ from Eq. (22), we find that $\mathbf{B A}=\mathbf{I}$. Thus, the dot product of row $n$ of $\mathbf{B}$ with column $\mathbf{n}$ of $\mathbf{A}$ is 1.0 and the dot product of row $n$ of $\mathbf{B}$ with all of the other columns of $\mathbf{A}$ is 0.0 . In general, only a few of the elements of column $\mathbf{n}$ of $\mathbf{A}$ are not equal to 0.0 and the nonzero elements are all equal to 1.0. For our example, column 52 has 4776 components and only 48 of the values are not equal to zero. We can approximate $\mathbf{B}$ by assuming that all of the elements in row $\mathbf{n}$ are equal to zero except the traces that correspond to the positive values in column $n$ of $\mathbf{A}$ and that all of the nonzero values in row $n$ of $\mathbf{B}$ are equal to the same value $\left(\rho_{n}\right)$. With this assumption, the dot product of row $n$ of $\mathbf{B}$ with column $n$ of $\mathbf{A}$ is $M_{n} \rho_{n}$, where $M_{n}$ is the number of nonzero values column $n$ of $A$. Since the dot product should be equal to 1.0 , the value of $\rho_{n}$ is: $=1 / M_{n}$. For our example, $M_{n}=48$ and $\rho_{\mathrm{n}}=0.0208$. In Fig. 2, the large values are approximately equal to 0.0208 .

In the last paragraph, we have derived the following approximate map from $\mathbf{v}$ to $\mathbf{x}$ :

$$
\mathrm{x}_{\mathrm{n}} \cong \frac{\sum_{1} \mathrm{a}_{\mathrm{n}} \mathrm{v}_{\mathrm{t}}}{\mathrm{Mn}_{\mathrm{n}}}
$$

Thus, we calculate $x_{n}$ by averaging over all of the components of $v$ that contain $x_{n}$.

We conclude this subsection by discussing the relationship between the decoupled small problems and the original large problem (see Fig. 3). We will iterate between the small problems and the large problem. For each iteration, the input to the method is an initial value in the $\mathrm{x}$ coordinates $\left[\mathrm{x}_{0}\right]$ which is mapped to initial values for the $\mathrm{v}$ coordinates $\left[{ }^{k} \mathbf{v}_{0}\right]$. Each of the decoupled global optimization problems is solved to determine $\left[{ }^{k} \mathbf{v}_{d}\right]$ 


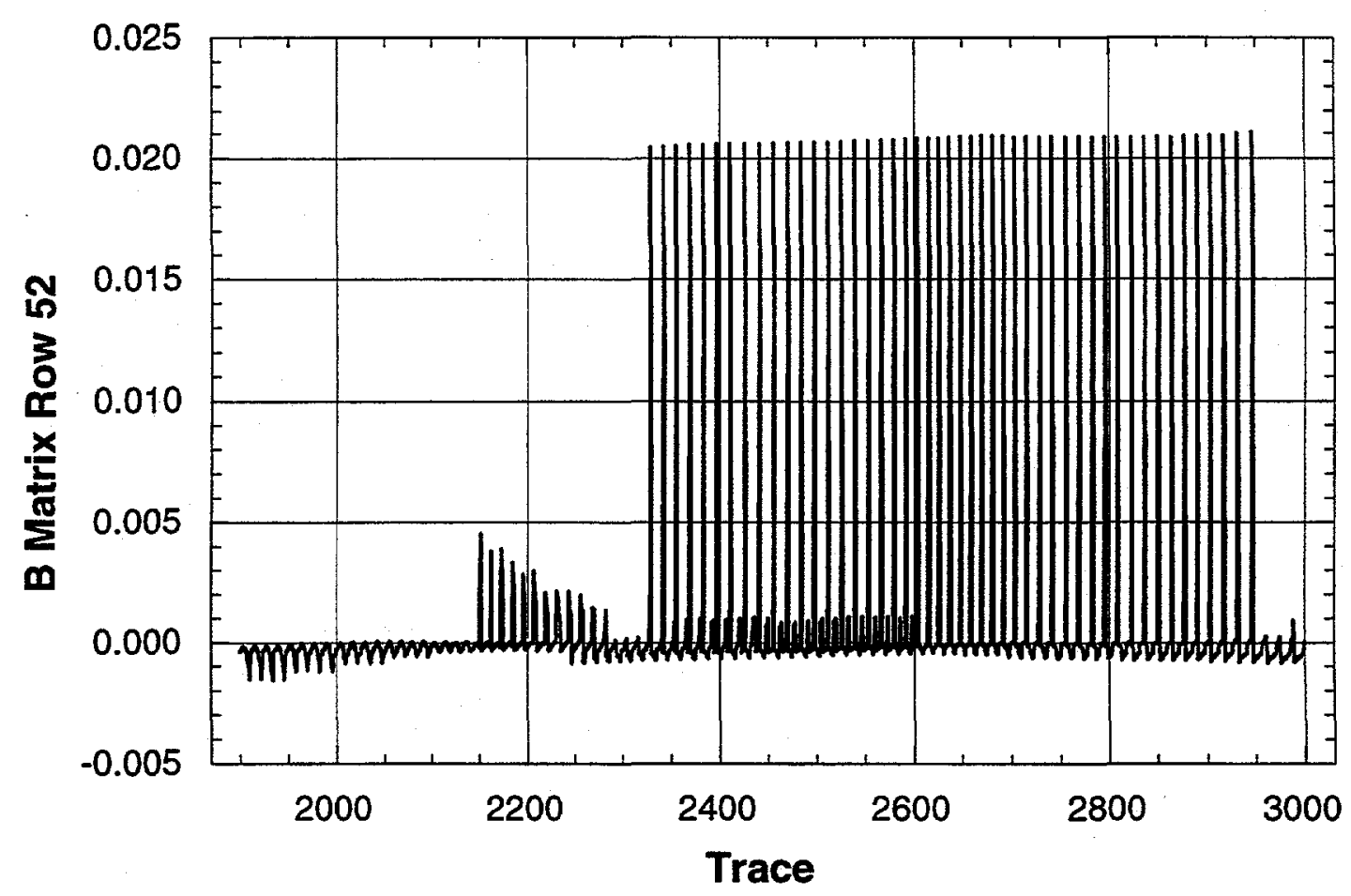

Fig. 2. Row 52 of the B matrix for the example problem.

and the maximum value for each component of the stack power for the decoupled problem $\left[{ }^{D} G_{k}\right]$. The $v$ coordinates are mapped back to the $\mathrm{x}$ coordinates to determine $\mathbf{x}_{d}$ which is used as an initial guess for the $\mathrm{N}$ parameter global optimization problem. The output of the method is the global maximum $\left[\mathbf{x}_{\mathbf{g}}\right]$. We have found that the solution of the decoupled problem provides an excellent initial estimate for the parameters for the coupled problem.

\subsection{NULL SPACE FOR THE DECOUPLED PROBLEMS}

In Subsection 2.3, we found three null vectors for the coupled statics problem. For the decoupled problems, the three null vectors become a single vector. Adding a constant to either the $S_{S_{t}}$ or the $R_{t} t$, adds a constant to the $v_{t}$. For the third vector, adding position dependent terms adds a constant to the $v_{t}$. Thus, the only null vector for the decoupled problems has unity for each component.

\subsection{CONVERGENCE FACTOR}

The convergence factor $\left(\mathrm{F}_{\mathrm{k}}\right)$ is the ratio of the power for each CMP $\left(\mathrm{E}_{\mathrm{k}}\right)$ and the decoupled power bound $\left({ }^{D} \mathrm{G}_{\mathrm{k}}\right)$ :

$$
\mathrm{F}_{\mathrm{k}}=\mathrm{E}_{\mathrm{k}} / \mathrm{D}_{\mathrm{k}}
$$




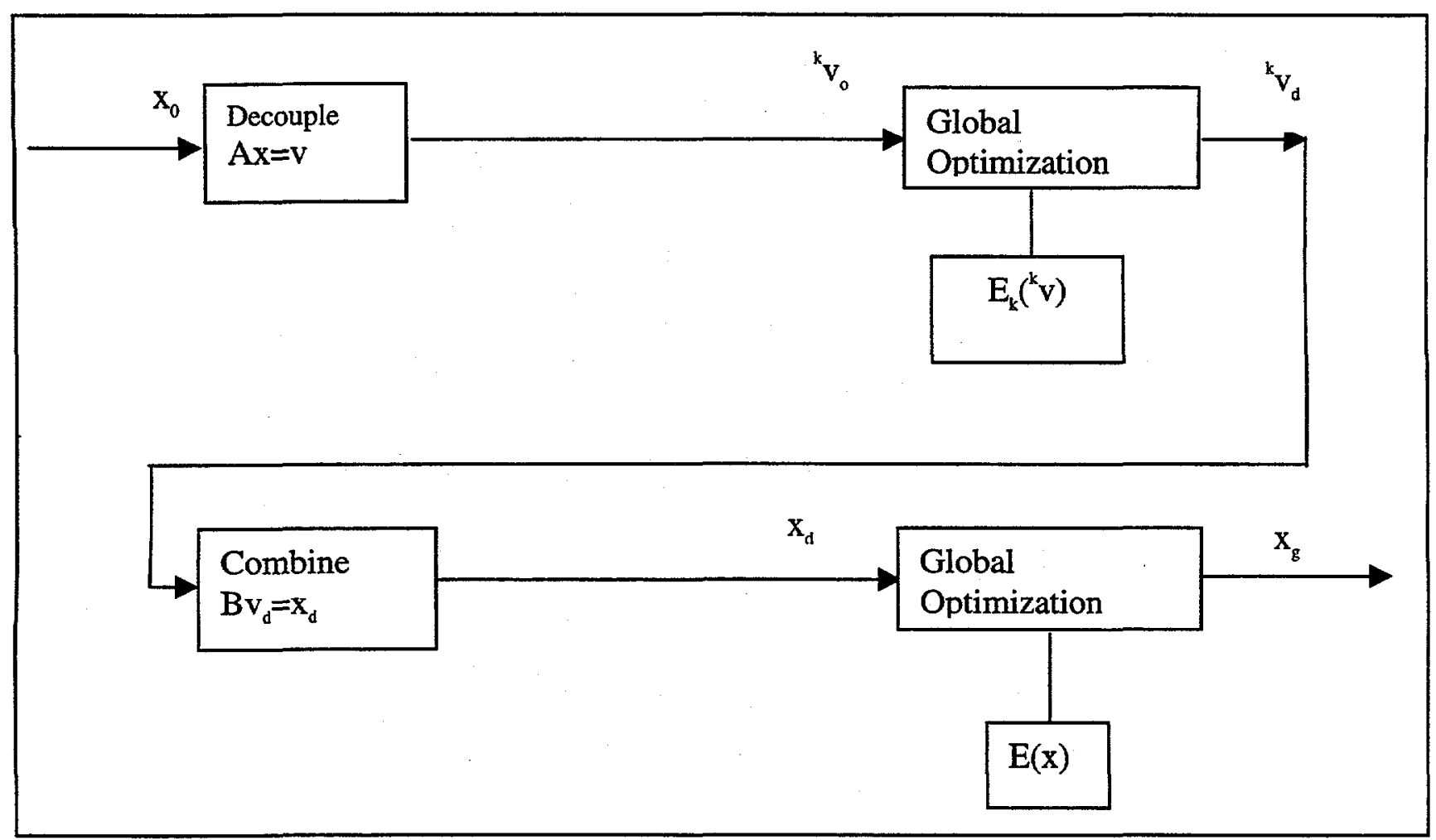

Fig. 3. The mapping between the decoupled problems and the large problem.

Since the $E_{k}$ are positive, each of the convergence factors will be in the range $(0,1)$.

\subsection{POWER NORM}

We will find many parameter vectors that give high values for the stack power. We want to know if the vectors are significantly different. We will use two norms to compare vectors: a modified Euclidean norm and a stack power norm. For the modified Euclidean norm, we remove the null space components before we calculate the Euclidean norm. We define a power norm by comparing the differences in stack power between two vectors.

We expect tradeoffs; the differences in the total power for two vectors might be small, while there could be large differences in the detailed components of the stack power. We want to compare the power for two vectors: ${ }^{c} \mathbf{x}$ and ${ }^{d} \mathbf{x}$. The power for the two vectors is ${ }^{\mathrm{c}} \mathrm{E}$ and ${ }^{\mathrm{d}} \mathrm{E}$ :

$$
{ }^{\mathrm{c}} \mathrm{E}=\sum_{\mathrm{k}}{ }^{\mathrm{c}} \mathrm{E}_{\mathrm{k}} \quad \text { and } \quad{ }^{\mathrm{d}} \mathrm{E}=\sum_{\mathrm{k}}{ }^{\mathrm{d}} \mathrm{E}_{\mathrm{k}}
$$

The power norm $\left(\rho_{\mathrm{cd}}\right)$ is the sum of the absolute differences of the components of the power for the two vectors:

$$
\Delta_{c d}=\sum_{k}\left|E_{k}-{ }^{d} E_{k}\right|
$$




\section{GLOBAL OPTIMIZATION ALGORITHM}

ORNL has developed a state-of-the-art algorithm for global optimization: TRUST. TRUST solves the following optimization problem: given an objective function $[\mathrm{f}(\mathbf{x})$ where $\mathbf{x}$ is an $\mathrm{N}$ dimensional vector that is constrained to lie in a domain (P)], find the global minimum $\left(\mathbf{x}_{\mathrm{g}}\right)$. That is, find a point $\mathbf{x}_{\mathrm{g}}$ in $P$ such that $f\left(\mathbf{x}_{\mathrm{g}}\right) \leq f(\mathbf{x})$ for all $\mathbf{x}$ in $P$. Since the goal in the statics problem is to maximize the total stack power $(E)$, the objective function for TRUST is: $f(x)=-E$. Several papers have been published that describe the deterministic version of TRUST [see Cetin (1993), Barhen (1996), and Barhen (1997)]. Recently, substantial enhancements have been incorporated into the TRUST algorithm. Here, we will only highlight some of these innovations. A more detailed discussion of the most recent improvements can be found in Oblow (1999).

In our initial approach to the statics problem, we used a deterministic version of TRUST that found the global minimum by executing a series of cycles of tunneling and descent. As discussed in Barhen (1997), TRUST begins at one corner of the hyperparallelpiped P. From the initial point, TRUST takes a small step into the interior of $P$ in the $\mathbf{p}$ direction. If $\mathbf{f}(\mathbf{x})$ is lower at the second point than at the first point, TRUST will descend to a local minimum. Otherwise, TRUST will tunnel following a curved path until it reaches a new basin of attraction (where $f(x)$ is lower than the current candidate for global minimum) or $\mathbf{x}$ leaves the region P. From each local minimum, TRUST takes a small step in the $\mathbf{p}$ direction and begins to tunnel. The TRUST algorithm terminates when $\mathbf{x}$ leaves the region $P$.

Two enhancements to TRUST are described in Barhen (1997): reflection of the path at the boundary of $\mathbf{P}$ and one-dimensional tunneling paths. Later in this section, we will discuss the one-dimensional tunneling paths.

A rigorous proof had shown that the deterministic version of TRUST would find the global minimum for the one-dimensional case (where $\mathbf{x}$ is a scalar). If we approximate an $\mathrm{N}$-dimensional problem by a $1 \mathrm{D}$ curve that covers the $\mathrm{N}$-dimensional (ND) region $\mathrm{P}$ (or by a regular grid), we can formally solve all global optimization problems. However, this method is not practical for large problems because the number of function evaluations increases exponentially with the number of dimensions (the number of evaluations is $M^{\mathrm{N}}$, where $M$ is the number of function evaluations in each dimension).

While the deterministic version of TRUST has been very successful in solving standard non-convex benchmark problems that have up to 20 parameters, it does not always find the globally optimum values for large residual statics problems with 100 or more parameters. The basic reason that TRUST may be less successful with large problems is that the size of the problem increases exponentially with the number of parameters. We will use three examples to illustrate this point. The first example was discussed in the last paragraph; we found that a comprehensive search of a regular grid in an ND space requires $\mathbf{M}^{\mathrm{N}}$ function evaluations. For the second example, we recall that TRUST begins at one corner of the hyperparallelpiped $\mathrm{P}$. For an ND problem, there are $2^{\mathrm{N}}$ corners where 
TRUST could start. In general, TRUST would explore a different path from each of the corners. The final example is that in a large dimensional space, a curved path has a smaller chance of cutting the basin of attraction of the global minimum. Consider a region $\left(\mathrm{P}^{*}\right)$ with each dimension equal to one half of the same dimension for $\mathrm{P}$. Then, the ratio of the volumes of $\mathrm{P}^{*}$ and $\mathrm{P}$ is $1 / 2^{\mathrm{N}}$. As $\mathrm{N}$ becomes large, the volume of the region $P *$ becomes a very small fraction of the total volume of $P$.

Since the deterministic version of TRUST does not always find globally optimum values for large residual statics problems, we enhanced TRUST by using one-dimensional tunneling paths [see Barhen (1997)]. From a local minimum, we use the 1D version of TRUST to explore each of the $\mathrm{N}$ dimensions of the problem one at a time. When we find a point where the objective function has a lower value than the previous local minimum, we descend to the next local minimum. If we have explored all of the $\mathrm{N}$ directions and have not found a lower value, we stop the algorithm. For the 1D searches, we can use a uniform grid or the nonuniform grid that results from the terminal repeller and the subenergy tunneling transformation. The choice of the dimension to search next can be numerical order or random.

Using the 1D tunneling paths allowed us to find much better values for the stack power. Subsequently, Oblow developed SPT (Stochastic Pijavskij Tunneling) that greatly speeds up the 1D tunneling phase and has found many large values for the stack power. We will describe SPT in the remainder of this section.

For the residual statics optimization problem, calculation of the stack power function $[f(x)]$ is expensive (requires many floating point operations) and the calculation of the derivatives of the function with respect to each of the components of the parameter vector $(\mathbf{x})$ is very expensive. During the descent phase of TRUST, derivatives are required to find a local minimum. While finding a local minimum is relatively expensive, the benefit is large. When we perform a search and find a lower value than the previous local minimum, we know that we are in the basin of attraction of a new local minimum that will be lower than the previous local minimum. Thus, we continually descend toward the global minimum. An algorithm that descends from many random starting points can repeatedly descend into a previously identified local minimum. By searching for a lower value than the previous local minimum, we gain two benefits. We do not need to calculate derivatives while searching and we only calculate derivatives when they will guide us to a new and lower local minimum.

The key parameter in the SPT algorithm is a pseudo-Lipschitz constant $\left(\mathrm{L}_{\mathrm{ps}}\right)$ that is used to define unpromising regions in the search space that do not need to be explored. For the 1D case (where the parameter vector is a scalar), the Lipschitz constant ( $L$ ) is an upper bound on the rate of change of the objective function [f(x)]:

$$
\left|\frac{\mathrm{df}}{\mathrm{dx}}\right| \leq \mathrm{L}
$$


The pseudo-Lipschitz constant $\left(\mathrm{L}_{\mathrm{ps}}\right)$ is the largest slope of any line drawn from the global minimum that is tangent to the curve that defines the basin of attraction for the global minimum (see Fig. 4). In general, the pseudo-Lipschitz constant is less than the Lipschitz constant. For a square well (a function that is constant before point a, decreases to a lower value at point $a$ and remains at the lower value to point $b$ at which it returns to the original value), the Lipschitz constant is infinity while the pseudo-Lipschitz constant is finite (if we assume that the location of the global minimum is at the midpoint between $a$ and $b$ ).

We will use Fig. 4 to discuss a simple version of the SPT algorithm for the 1D case. We evaluate the function at point $1\left(x_{1}\right)$. From $x_{1}$, we descend to the local minimum $\left(f_{1}\right)$. The local minimum is our best candidate for the global minimum: $f^{*}=f_{1}$. From $x_{1}$, we draw a line (half of a Pijavskij cone) that has the same slope as the pseudo-Lipschitz line. The intersection of the line from $x_{1}$ and the line $\left(y=f_{1}\right)$ determines a small region of the $x$ axis (the left shaded region on the $f_{1}$ line in Fig. 4) that cannot have a lower value than the current minimum value $\left(f_{1}\right)$.

We choose a second point in the available portion of the $\mathrm{x}$ axis $\left(\mathrm{x}_{2}\right)$ and evaluate the function. Since $f\left(x_{2}\right)$ is greater than $f^{*}$, we are tunneling (and we do not descend to a local minimum). From $x_{2}$, we draw a Pijavskij cone (the magnitudes of the slopes of the two lines are equal to the slope for the pseudo-Lipschitz line). The intersection of the cone from $x_{2}$ and the $f_{1}$ line excludes a small region of the $x$ axis.

We choose a third point in the available portion of the $\mathrm{x}$ axis $\left(\mathrm{x}_{3}\right)$ and evaluate the function. Since $f\left(x_{3}\right)$ is less than $f^{*}$, we are in the basin of attraction of a new local minimum. From $x_{3}$, we descend to the local minimum $\left(f_{2}\right)$ and $f^{*}=f_{2}$. From $x_{3}$, we draw a Pijavskij cone. Since the current local minimum is much lower than $f_{1}$, the three Pijavskij cones now exclude much more of the $\mathrm{x}$ axis. We choose a fourth point in the available portion of the $x$ axis $\left(x_{4}\right)$ and evaluate the function. Since $f\left(x_{4}\right)$ is greater than $\mathrm{f}^{*}$, we are tunneling. From $\mathrm{x}_{4}$, we draw a Pijavskij cone that excludes a large region of the $\mathrm{x}$ axis.

We choose a fifth point in the available portion of the $\mathrm{x}$ axis $\left(\mathrm{x}_{5}\right)$ and evaluate the function. Since $f\left(x_{5}\right)$ is less than $f^{*}$, we are in the basin of attraction of a new local minimum. From $x_{5}$, we descend to the local minimum $\left(f_{3}\right)$ and $f^{*}=f_{3}$. We evaluate the function at a few more points and conclude that $f_{3}$ is the global minimum for the example in Fig. 4.

We have found that the Pijavskij cones exclude more of the $\mathrm{x}$ axis as the current best local minimum ( $f^{*}$ ) decreases. We can improve the efficiency of the SPT algorithm by adding a second parameter: an estimate of the global minimum $\left(\mathrm{f}^{\mathrm{G}}\right)$. In the early stages of the algorithm, $\mathrm{f}^{\mathrm{G}}$ will increase the region on the $\mathrm{x}$ axis that is excluded by the Pijavskij cones. At the later stages of the algorithm, the current candidate for the global minimum (f*) may become lower than $f^{G}$ and the parameter will have a small impact. In Subsection 


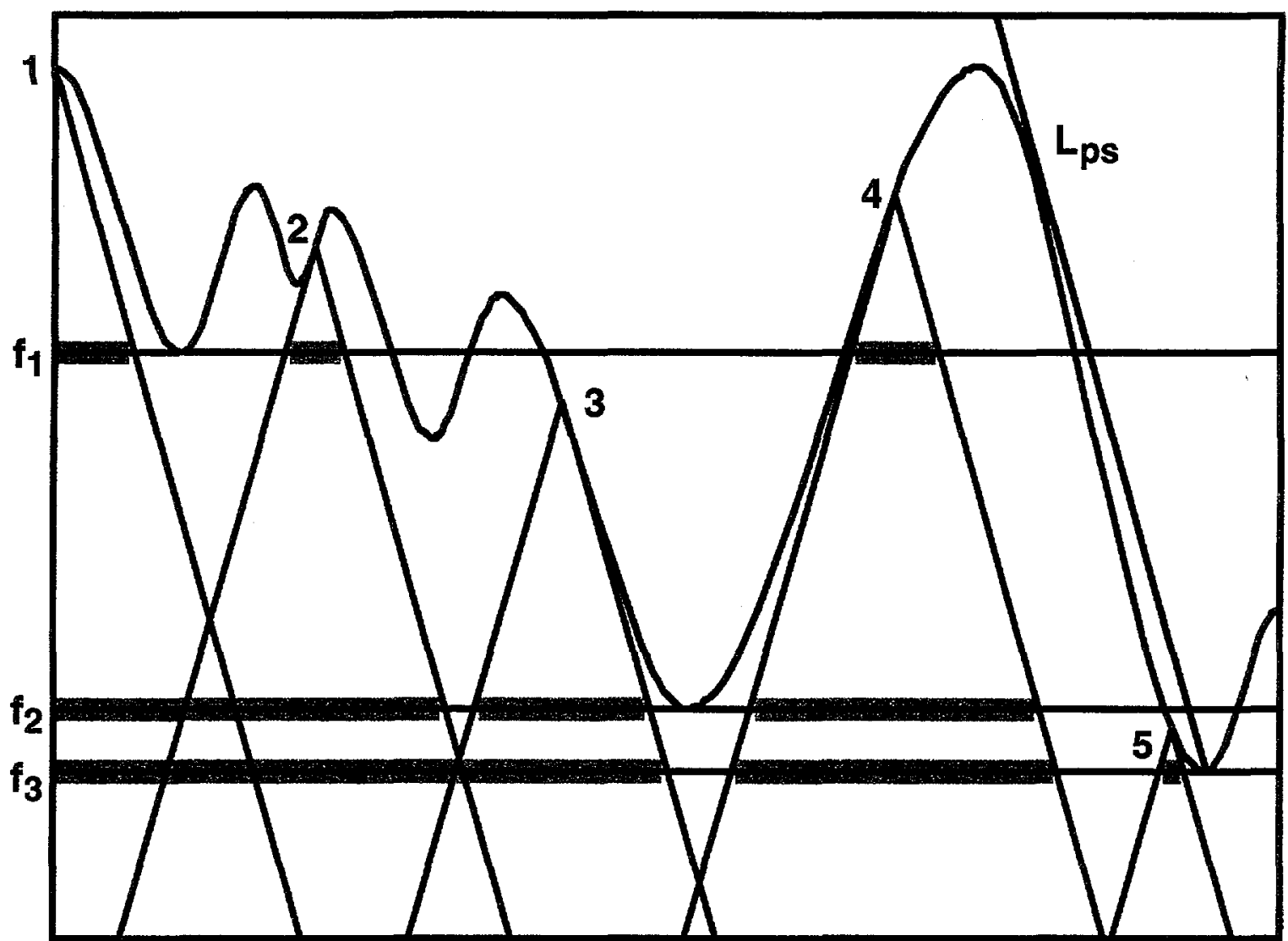

Fig. 4. Finding the global minimum for a 1D example using the SPT algorithm.

2.4, we derive a close upper bound for the stack power that can be used to estimate $f^{G}$. After several attempts at finding the global minimum, we can use the best value found to date as an estimate of $\mathrm{f}^{\mathrm{G}}$.

The resolution is the width of the smallest basin of attraction that can be detected. For the searches that we will discuss in the results section, we have assumed that the range of each component of $x$ was $\pm 50 \mathrm{~ms}$ (for a total range of $100 \mathrm{~ms}$ ). Since we have used a resolution that allowed 100,000 points in the range, our resolution is $1.0 \mu \mathrm{s}$. For a comparison, Rothman (1986) had a range of $\pm 160 \mathrm{~ms}$ and a resolution of $8 \mathrm{~ms}$, while DuBose (1993) had a range of $\pm 50 \mathrm{~ms}$ and a resolution of $2 \mathrm{~ms}$. While our resolution is more than three orders of magnitude better than the previous work, there is no physical justification for having a resolution that is greater than a few samples within one cycle of the highest frequency that is in the data set. If the highest frequency is $60 \mathrm{~Hz}, 2 \mathrm{~ms}$ is a reasonable resolution. Our method allows us to work at a much higher resolution than can be justified by the experimental details. We can imagine an objective function that is constant except for randomly distributed square wells of varying depth that are $1.0 \mu \mathrm{s}$ wide. For this function, the Pijavskij cones would not eliminate any regions of the $\mathrm{x}$ axis and we would need to evaluate the objective function 100,000 times to find the global minimum. For the problem that we shall discuss in the results section, the Pijavskij cones quickly eliminate all of the $\mathrm{x}$ axis and we usually evaluate the function about ten times to 
achieve a resolution of 100,000 points in the parameter range. Thus, we do not pay a penalty for working at high resolution.

The key parameter in the SPT algorithm is a pseudo-Lipschitz constant $\left(\mathrm{L}_{\mathrm{ps}}\right)$. We can use two complementary methods to estimate $\mathrm{L}_{\mathrm{ps}}$ : measure derivatives and set resolution. As we perform searches and descents, we calculate the function and the derivatives of the function with respect to each component of $\mathbf{x}$. We can monitor the derivatives and determine the largest values. The pseudo-Lipschitz constant should be larger than any measured value. Consider a 1D search. The pseudo-Lipschitz constant is the ratio of a change in $f(x)$ to a change in $x$ [see Eq. (29)]. The change in $f$ is the difference between the current value of $f$ and our lower bound on $f$, while the change in $x$ is one half the resolution. Thus, our second parameter [an estimate of the global minimum $\left(f^{G}\right)$ ] and the resolution can be used to estimate the first parameter [the pseudo-Lipschitz constant]. Since our resolution is very small, we calculate much higher values for the pseudoLipschitz constant than we obtain by measuring derivatives.

We will outline a simple version of the SPT algorithm for the general case where $\mathbf{x}$ is an $\mathrm{N}$ dimensional vector.

1. Select a starting point. The values of the components of $\mathbf{x}$ could be: all zero, from the disaggregated problem [from Eq. (22)], or random numbers.

2. Use a descent algorithm to find a local minimum.

3. Begin a loop over the $\mathbf{N}$ components of $\mathbf{x}$. For each loop, randomly choose an integer (n) in the range 1 to $\mathrm{N}$.

4. Perform a 1D SPT search (as outlined in Fig. 4) where all components of $\mathbf{x}$ are constant except $\mathrm{x}_{\mathrm{n}}$. If the search finds a point in a new basin of attraction, stop the search and descend to the next local minimum.

5. End of the loop that began in step 3. Stop if the loop has been performed $\mathbf{N}$ times. Otherwise return to step 3.

In more elaborate versions of the algorithm, we can perform the loop that begins at step 3 several times or begin the loop that starts at step 1 several times.

\section{RESULTS}

Our objective was to use TRUST to estimate surface-consistent residual statics. We have applied TRUST to several synthetic data sets. In all cases, TRUST attempted to solve the global optimization problem without using the values of the synthetic disrupting statics. The first (small) data set contained 24 shots and 50 receivers, for a total of 74 parameters. Although TRUST quickly solved the problems for the small data set, the values of the statics corrections were small and the solutions were found by descent from the initial point $(\mathbf{x}=\mathbf{0})$. The second (medium) data set was designed to be more of a challenge for TRUST with large statics corrections that cannot found by a descent from the initial point. The medium data set contained 77 shots and 77 receivers, for a total of 154 parameters. Several major changes in TRUST (that were discussed in Sections 2 and 3) 
were required to solve the problems with the medium data set. For the both the small and medium data sets, the coherence factors were large (near 1.0) and the increase in stack power was very large.

The third (large) data set was created by adding disrupting statics to measured seismic data. The large data set has 100 shots and 216 receivers, for a total of 316 parameters. The number of CMPs is 423 . The number of traces is 4776 and the number of frequencies is 118. Time required to calculate the stack power and the gradient of the stack power is 100 times longer than for the medium data set. The stack power at the initial point $(\mathbf{x}=\mathbf{0})$ was 882. The upper bound on the stack power [G see Eq. (14)] is :

$$
\mathrm{G}=\sum_{\mathrm{k}} \mathrm{G}_{\mathrm{k}}=6589 \text {. }
$$

We applied TRUST to the 423 decoupled problems. Most of the best values for the coherence factor were much less than 1.0. The total energy for the 423 decoupled problems $\left({ }^{\mathrm{D}} \mathrm{G}\right)$ is 2706 . Using Eq. (22), the $4776 \mathrm{v}$ coordinates were mapped back to the $316 \times$ coordinates to determine an initial point $\left(\mathbf{x}_{\mathrm{d}}\right)$ for the 316 parameter global optimization problem. The initial value for the energy was 1035. The first local maximum was 2183. After 98 iterations, an interim version of TRUST found the maximum value at 2366 .

After the development of the SPT version of TRUST, we have found many points with high values of the stack power. We will discuss a few (22) of the points with stack power greater than 2365 . In this section, we will display the seismic image that we were given and comparing it to the image after the application of residual statics corrections. We will discuss the coherence factors for our best solution. We will address the question: "Are the 22 points distinct?" using both a modified Euclidean distance norm and the power norm. We will compare our best values for the statics with the disrupting statics that were used to create the data sets.

We begin with three seismic images: before disruption, disrupted, and after correction. The original seismic image before the application of disrupting statics is displayed in Fig. 5. The disrupted seismic image is plotted in Fig. 6 while the image after the static corrections is shown in Fig. 7. The seismic reflections are well defined in Figs. 5 and 7 and not defined in Fig. 6. There are some interesting differences (cycle skips) between Figs. 5 and 7 that we will discuss later in this section. 


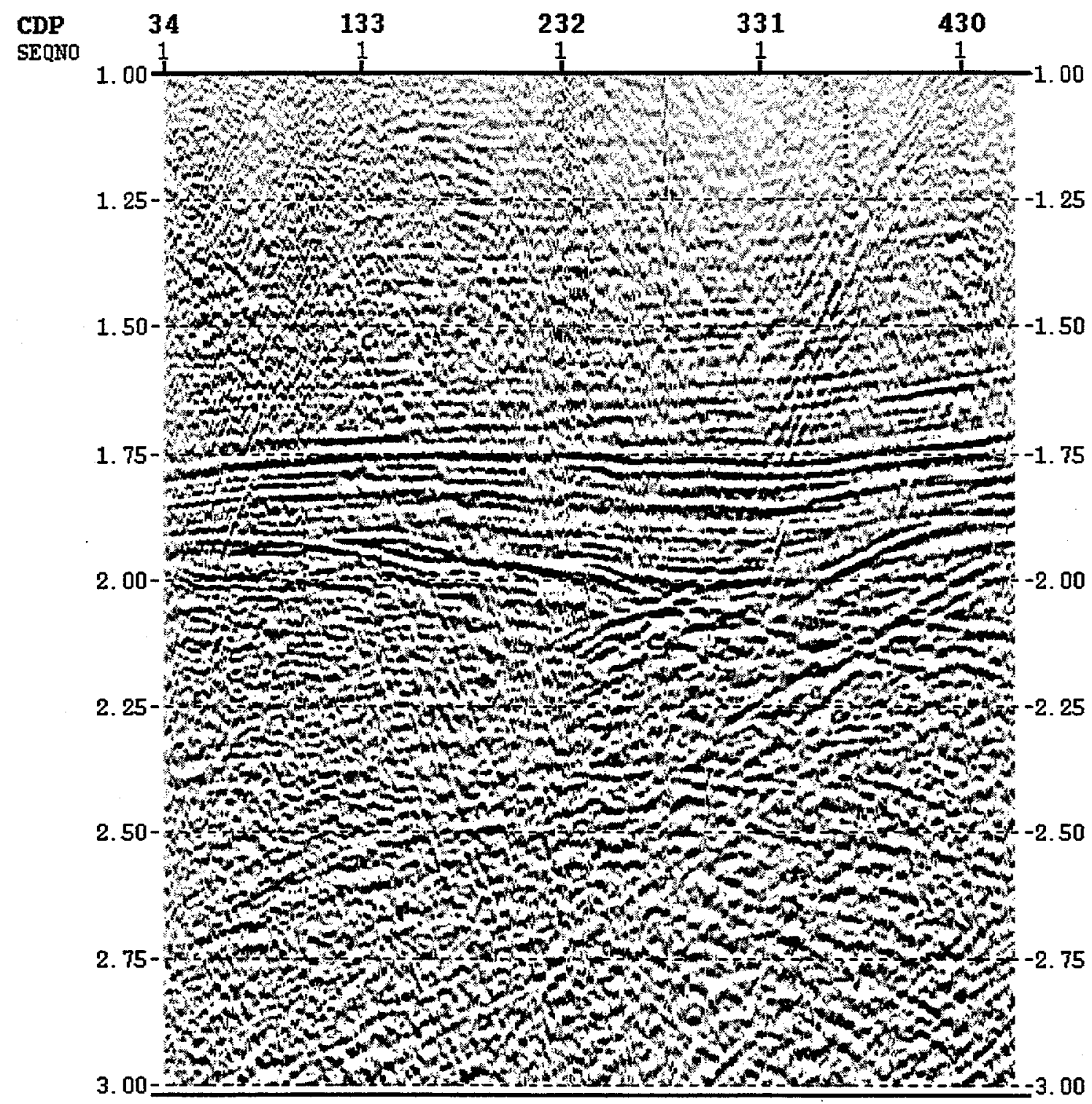

Fig. 5. The seismic image before the application of the disrupting statics.

In Section 2.2, we defined the coherence factor $\left(\mathrm{Q}_{\mathrm{k}}\right)$ as the ratio of the stack power $\left(\mathrm{E}_{\mathrm{k}}\right)$ for each CMP and the upper bound on the power $\left(\mathrm{G}_{\mathrm{k}}\right)$. In Fig. 8, we plot the stack power $\left(E_{k}\right)$ for the case when the total power is 2441 and the upper bound $\left(G_{k}\right)$ for each CMP. In most cases, the upper bound is much greater than the stack power. Since the upper bound on the stack power is 6589 , the weighted average value for the coherence factors is the ratio of 2441 and 6589: 0.370 . Thus, most of the traces are not in phase. 


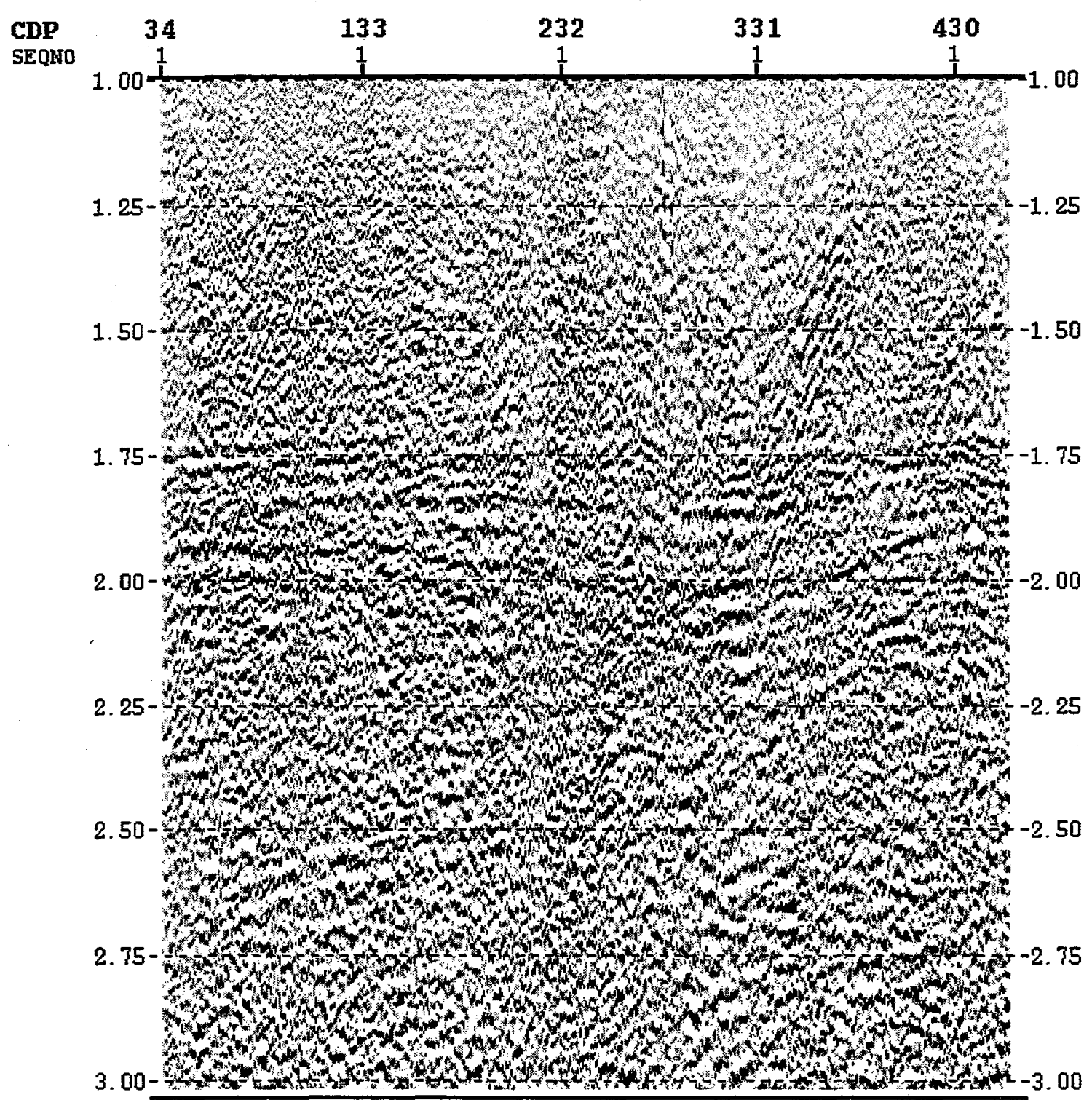

Fig. 6. The disrupted seismic image before the application of residual statics corrections.

In Section 2.4, we introduced new variables (v) that decouple the CMP gathers. Each of the decoupled global optimization problems can be solved independently using TRUST to find the stack power for the decoupled problem: ${ }^{D} G_{k}$. Using the ${ }^{D} G_{k}$, we can define a best (close upper bound for the) coherence factor (see Fig. 9). Most of the values for the best coherence factor range from $30 \%$ to $50 \%$. 


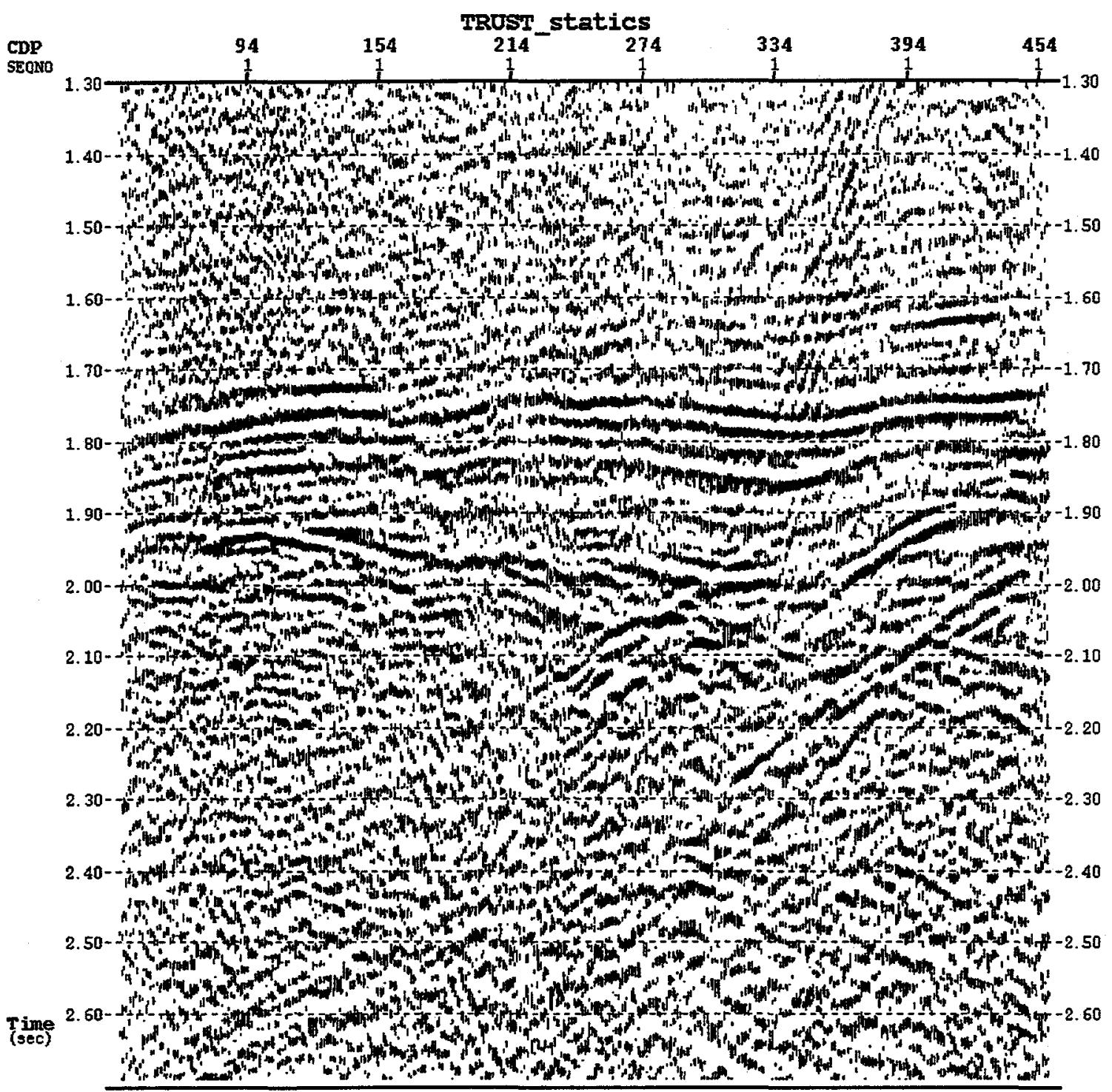

Fig. 7. The seismic image after the application of residual statics corrections.

We have developed two ways to compare our highest values for the stack power to the disaggregated upper bound on the stack power. The first is to subtract the current values for $E_{k}$ from the best values $\left(G_{k}\right)$. Since the best values are an upper bound, the differences will always be positive. The differences are plotted in Fig. 10 for two values of the stack power: 882 and 2427. When the power is large (2427), most of the differences are small (less than 4.0). 


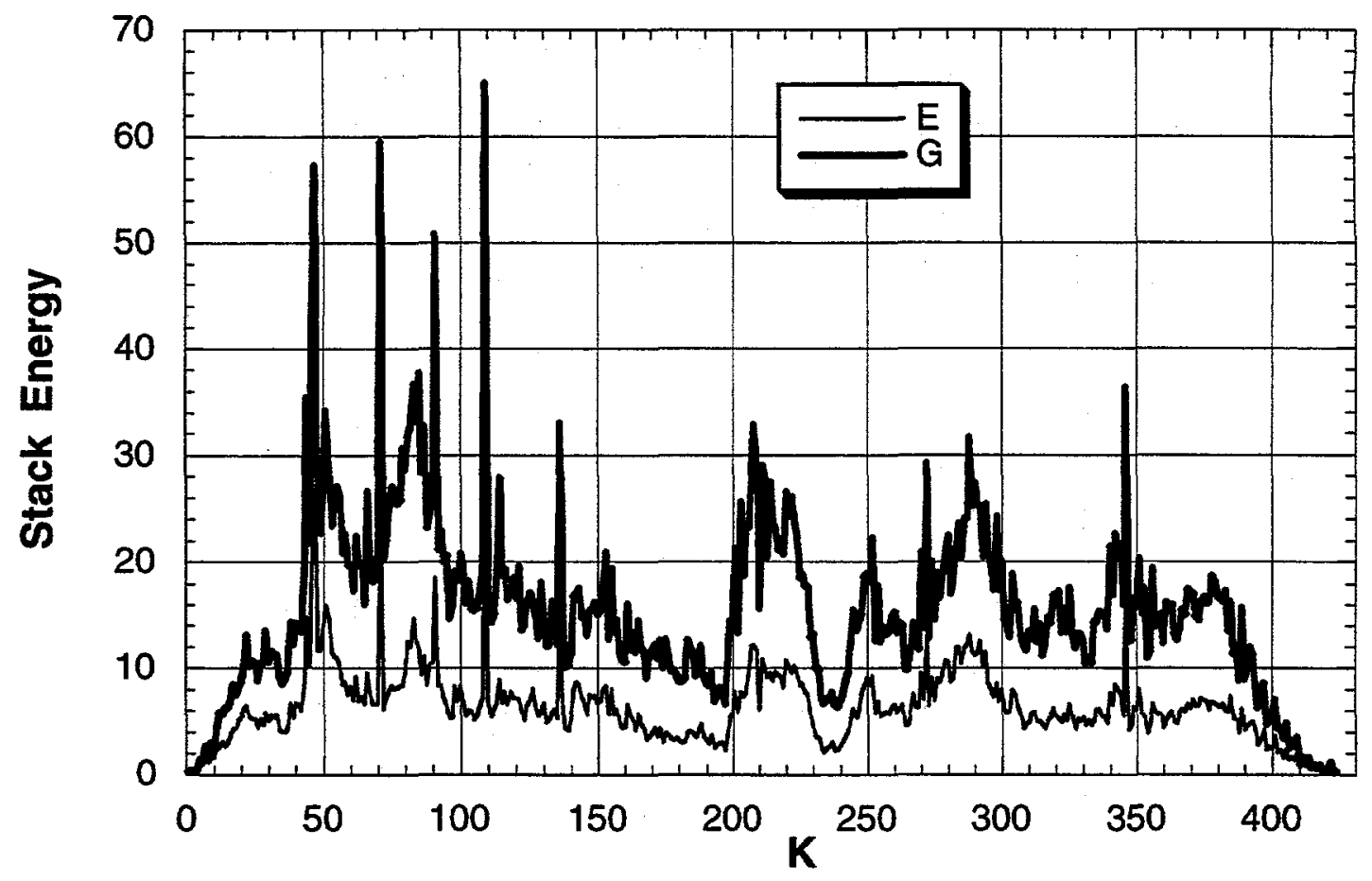

Fig. 8. The stack power $(E)$ and the upper bound $(G)$ for each CMP.

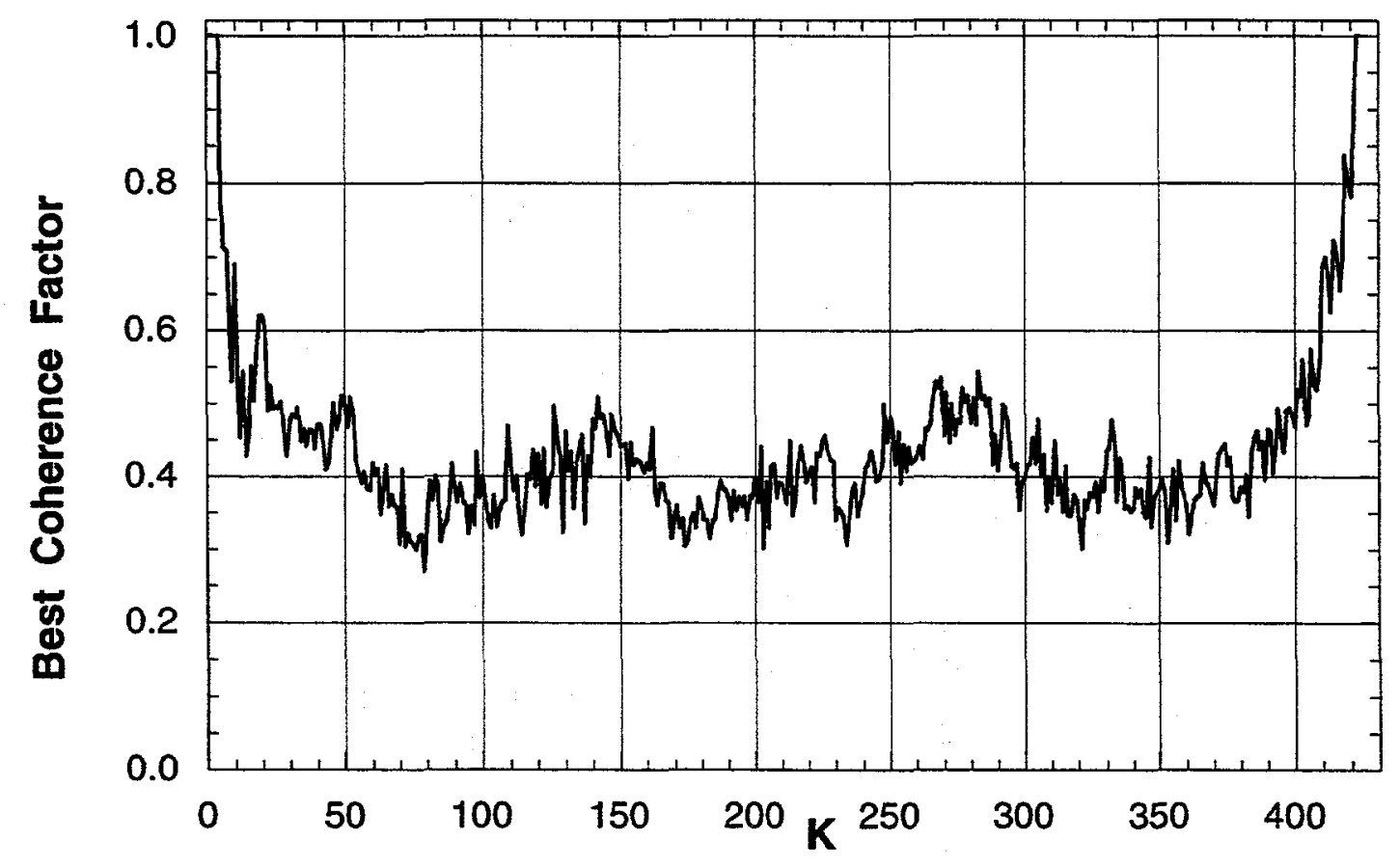

Fig. 9. The best coherence factor. 


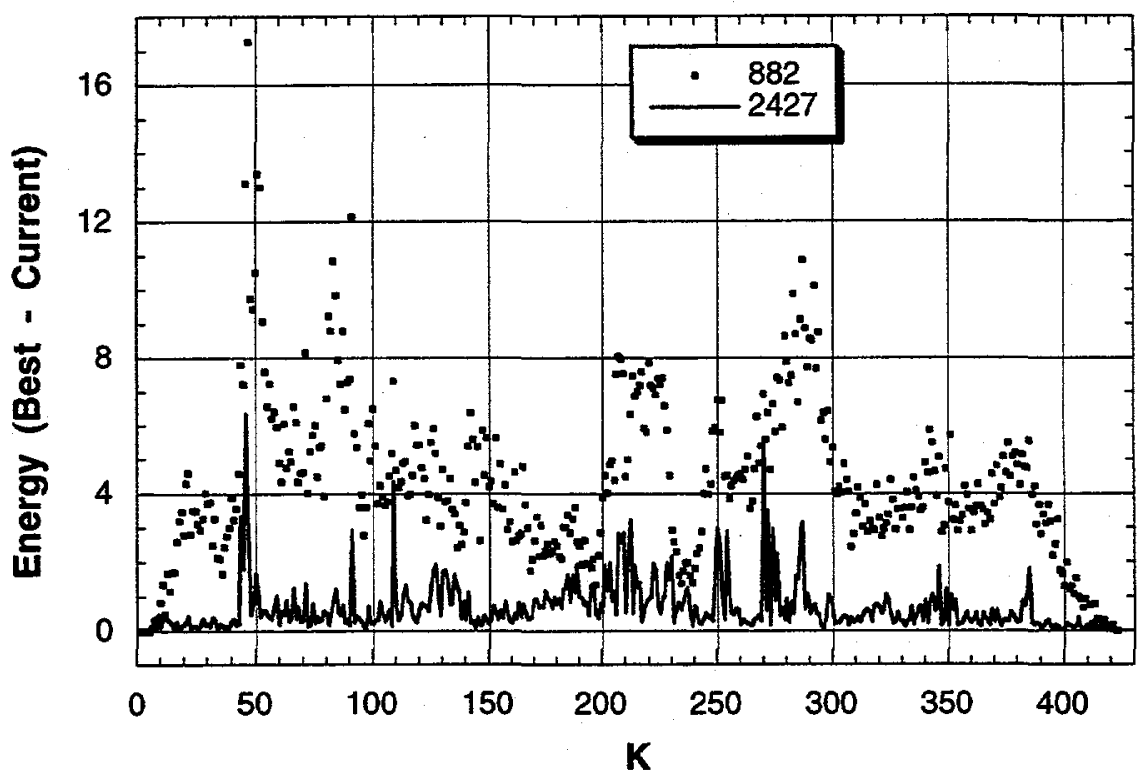

Fig. 10. The difference between the current value of the stack power and the best value for two cases: 882 and 2427 .

The second method is to use the convergence factor [the ratio of the power for each CMP $\left(E_{k}\right)$ and the decoupled power bound $\left.\left({ }^{D} G_{k}\right)\right]$ (see Fig. 11). In Fig. 11, most of the values for the convergence factor are above $80 \%$ when the power is large (2441).

After the development of the SPT version of TRUST, we have found many points with high values of the stack power. We will discuss a few (22) of the points with stack power greater than 2365 . We will address the question: "Are the 22 points distinct?" using both a Euclidean distance norm and the power norm defined in Section 2.7. To define the distance between two vectors, we find the difference between the vectors, subtract the three null space components and use the Euclidean norm on the residual difference. In Fig. 12, we plot the difference between each vector and all of the other vectors. The difference between vector one and vector 22 is displayed twice in the figure: once when $\mathrm{N}=1$ and once when $\mathrm{N}=22$. In Fig. 12, the differences ranged from 2.0 to 137.0 and only a few of the values are smaller than 20.0 .

In general, the null space correction is not very large. We will define the null space correction to be the difference in Euclidean norm for the distance between two vectors with and without the null space correction. In Fig. 13, the null space correction is plotted versus the corrected Euclidean norm for all of the differences between the vectors. All of the corrections were less than 12.0 (most are less than 8.0) and a few are 0.0.

In Fig. 14, we plot the power norm difference between each vector and all of the other vectors. The power norm differences range from 9.0 to 95.0. In Fig. 15, we plot the 
power norm vs the Euclidean distance. While none of the values are zero, there is a cluster of points in the lower left corner that are the smallest values for both power norm and Euclidean distance. Although there is a weak linear correlation between the power norm and Euclidean distance, there is a lot of scatter in the data and the value of $R^{2}$ is low $(0.22)$.

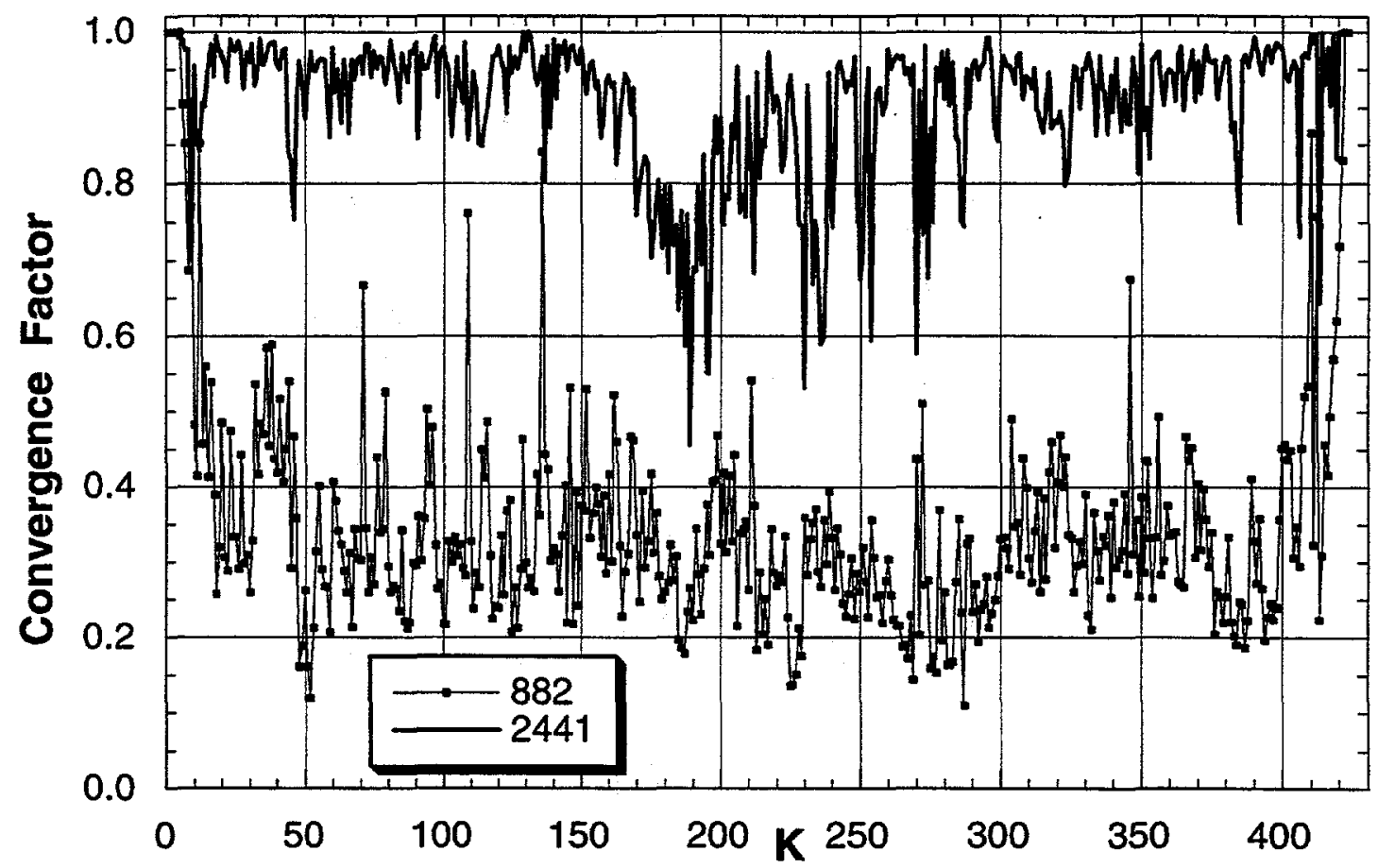

Fig. 11. The convergence factor for two cases: 882 and 2441.

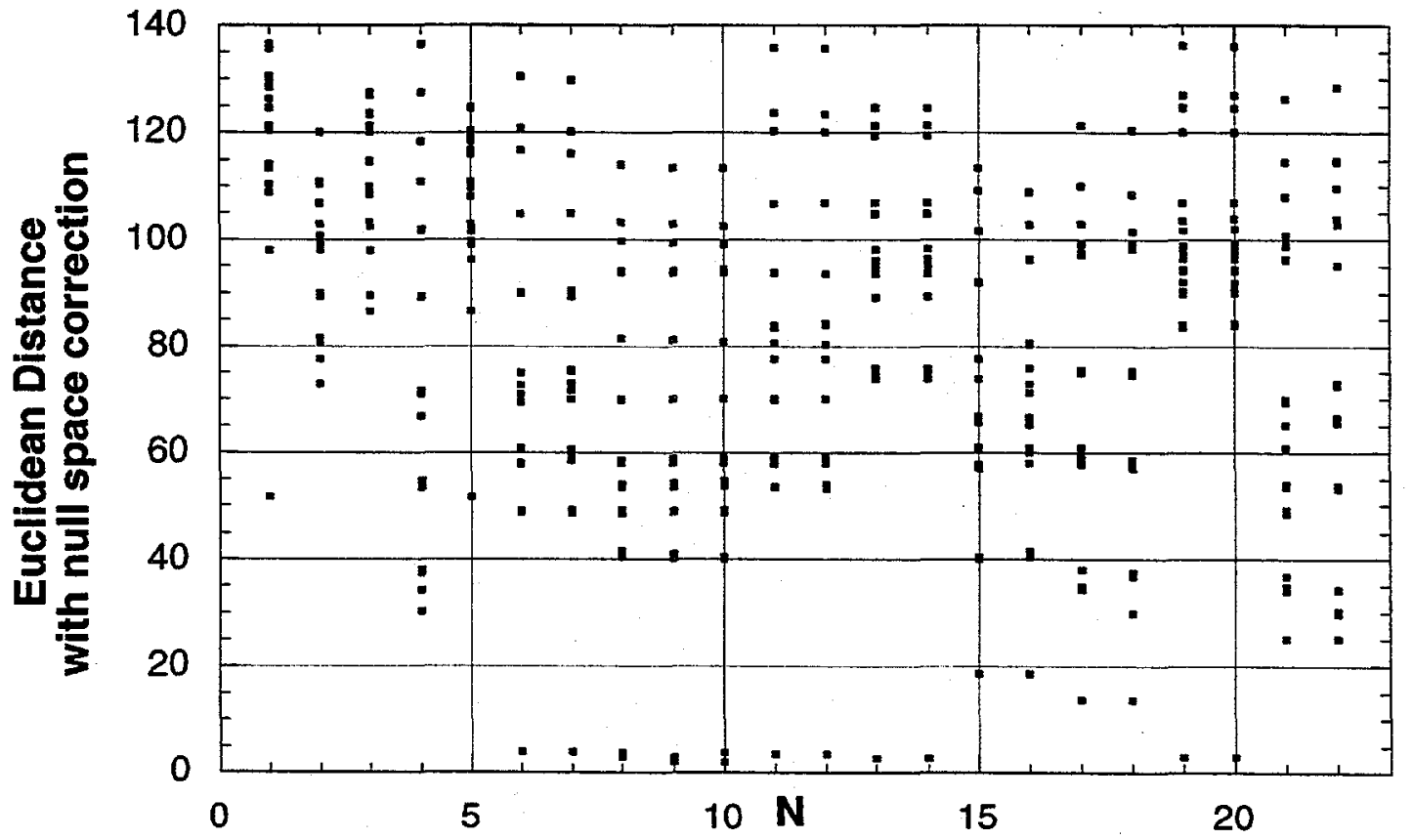

Fig. 12. The Euclidean distance between the 22 vectors after null space corrections. 


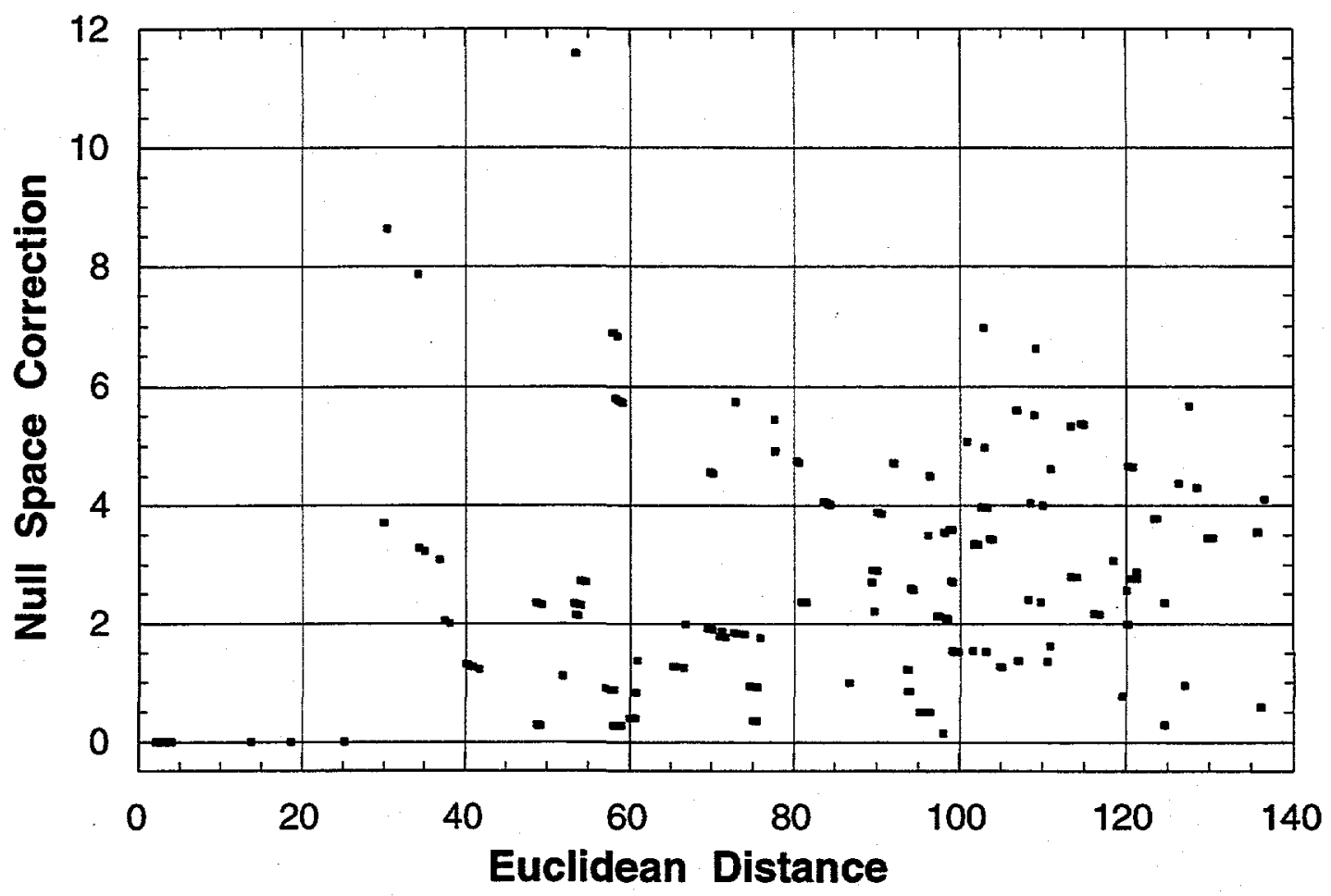

Fig. 13. The null space correction for the distances between the 22 vectors.

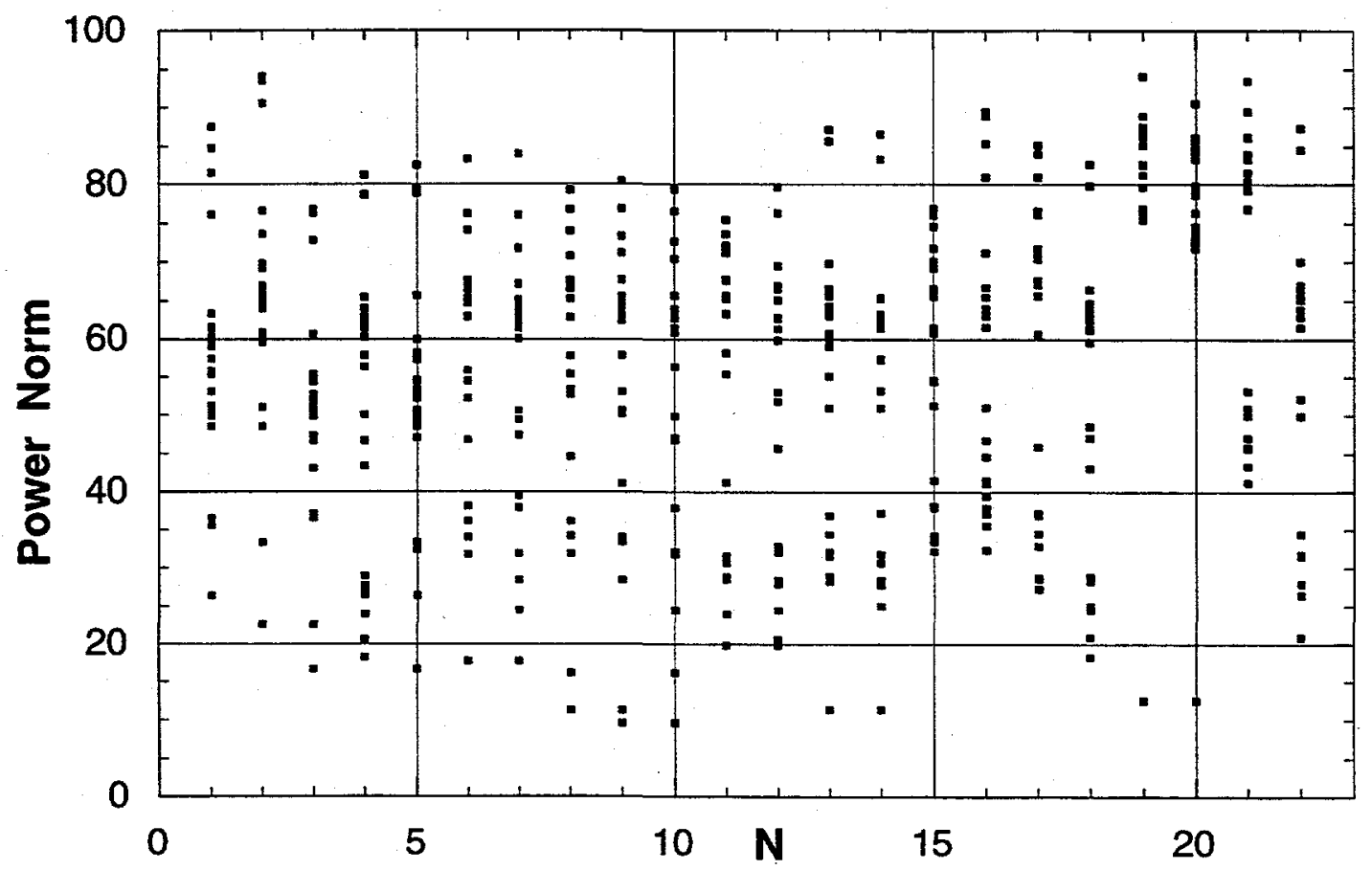

Fig. 14. The power norm distance between the 22 vectors. 


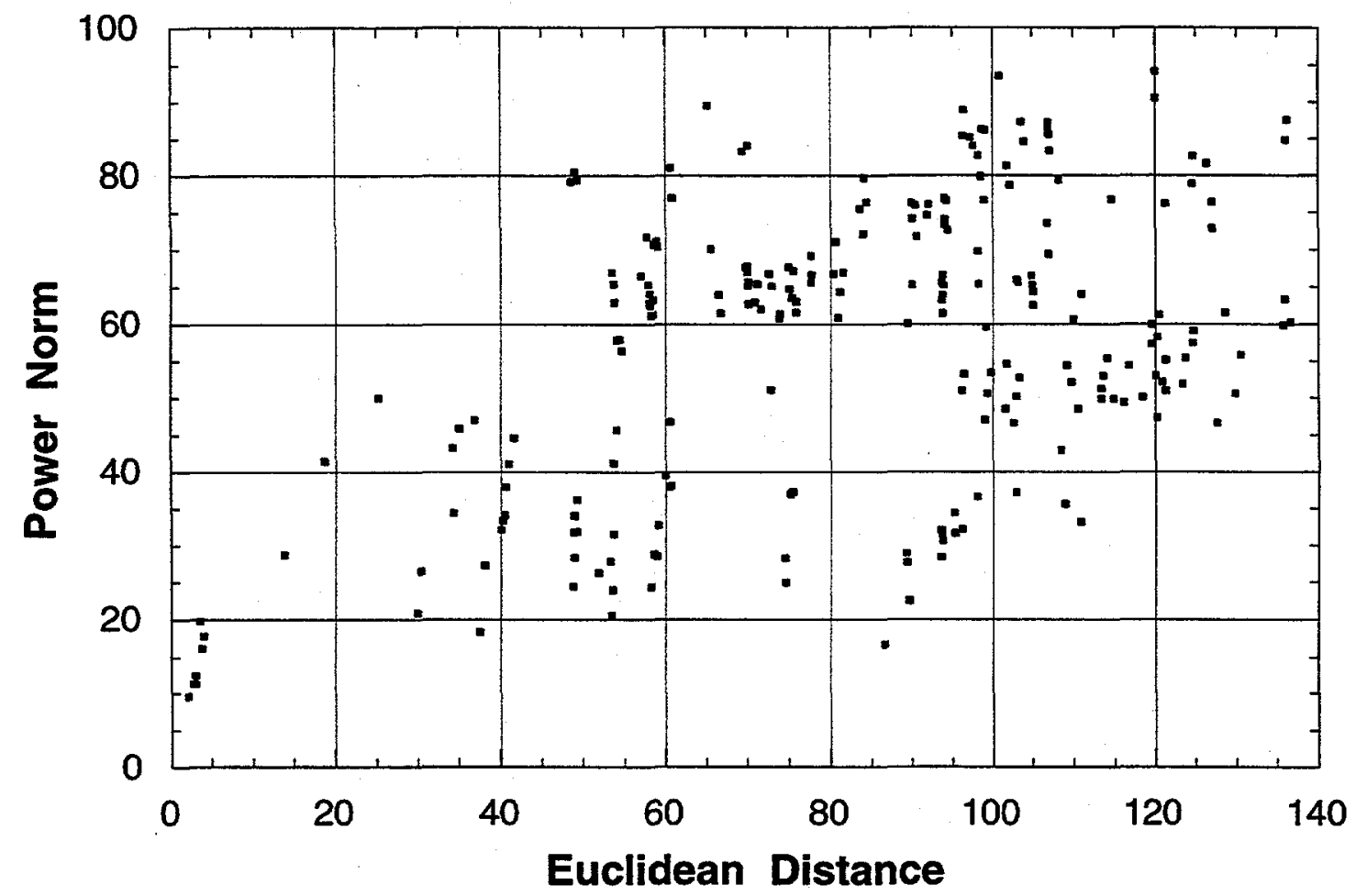

Fig. 15. Correlation between the power norm and Euclidean distance.

In Fig. 16, we display the Euclidean distance versus the stack power for each of the 22 cases. All of the small distances (less than 20.0) occur for the lower values of the power (less than 2405). For the two best values of the power (2427 and 2441), the lowest value of the distance is 72.8. Thus, the two best values are not close to any of the other points (and are not close to each other).

In Fig. 17, we plot the power norm versus the stack power for each case. Unlike the previous figure, each of the 22 cases has a substantial range in the power norm. We can understand the reason for the large range by defining another metric: the power difference. The power difference is the absolute value of the difference between the stack power for the two cases. We can show that the power difference is a lower bound for the power norm. When the power norm is equal to the power difference, every component of the stack power for one of the cases is greater than the corresponding component for the other case. The power difference between the highest power (2441) and the lowest power (2365) is 76. Thus, for the cases with either the smallest power or the largest power, the maximum value of the power norm will be greater than 76 . In Fig. 17, the largest value of the power norm is greater than 76 for all 22 cases.

In Fig. 18, we plot the power norm versus the power difference for the 22 cases. Since the difference is a lower bound for the norm, all of the points are above the 45 degree line (where the norm equals the difference). In Fig. 19, we display delta (the difference between the power norm and the power difference) versus the stack power. Delta decreases as the power increases. The small values of delta for the two highest cases 

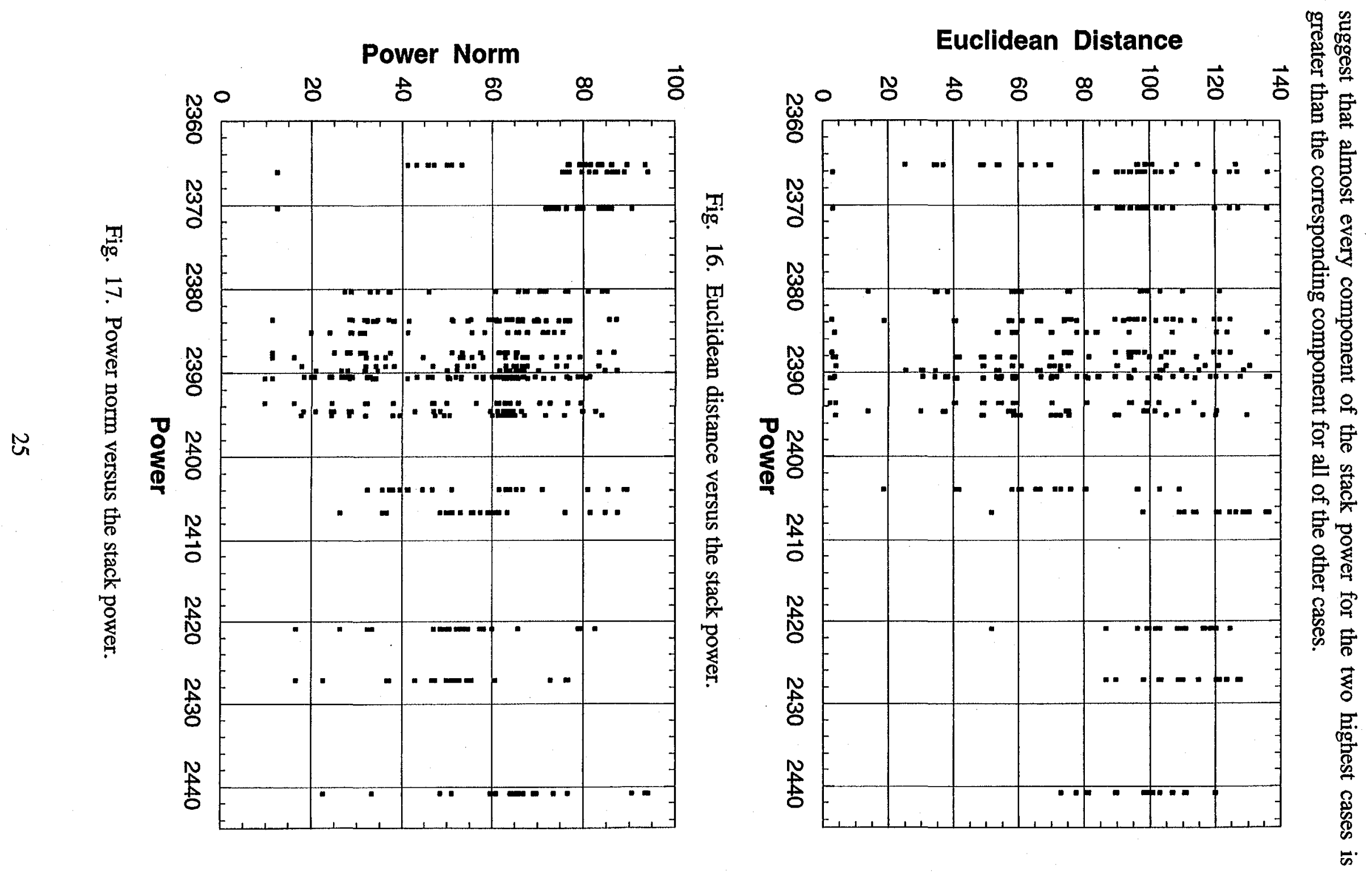


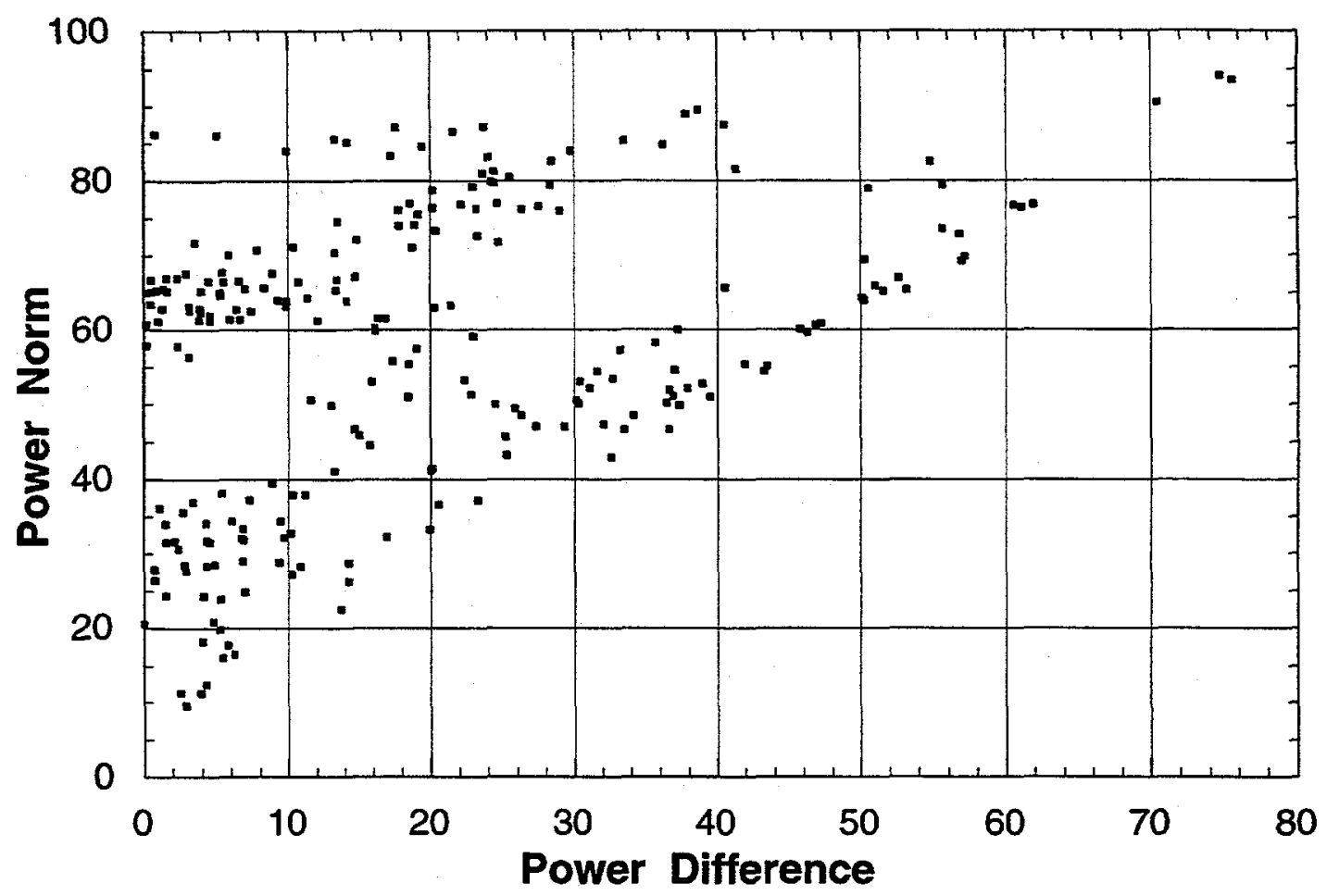

Fig. 18. Power norm versus the power difference.

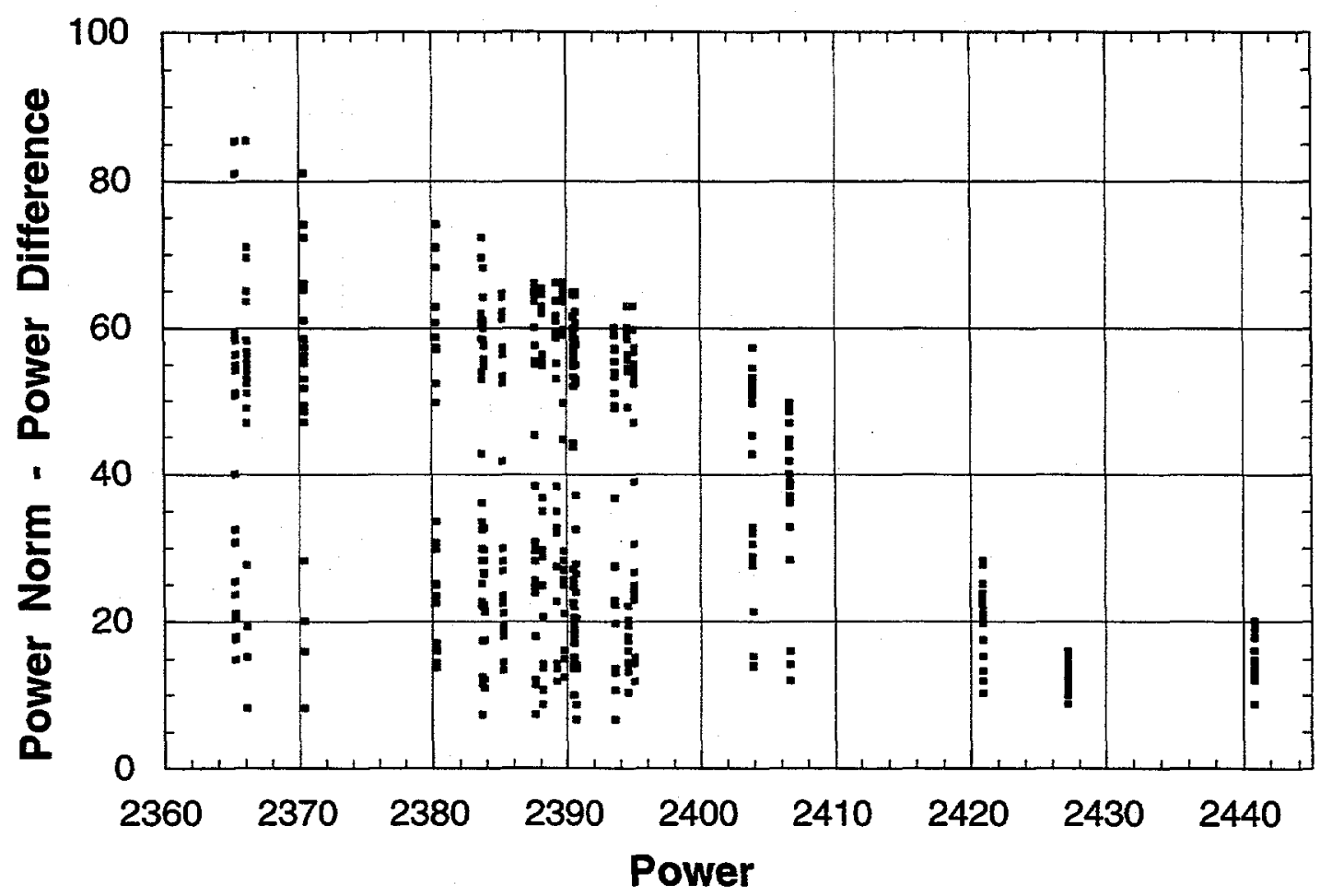

Fig. 19. The difference between the power norm and the power difference versus the stack power. 
After we solved the problem, we compared our estimates of the statics with the disrupting statics that had applied to the original seismic data (Fig. 5) to produce the input data for this project (Fig. 6). The disrupting statics are plotted in Fig. 20 and range from -21.0 to 24.0. To compare our results to the disrupting statics, we subtract the disrupting statics from our results. The differences are displayed in Fig. 21 for Case 2441 and in Fig. 22 for Case 2427. The most striking result is that five components of the $\mathbf{x}$ vector have large differences (cycle skips) for the two cases and the cycle skips occur for the same components $(83,160,201,276$, and 297). When we plot the difference between Case 2441 and Case 2427 in Fig. 23, we do not find cycle skips.

When we plot our solutions (Fig. 7), the lines that have strong reflections are not as continuous as the lines in Fig. 5. However, when we calculate the stack power for the disrupting statics in Fig. 20, we find that the power is 2349 (lower than the 22 cases). When we start from the disrupting statics and climb to the top of the first peak, we reach a power of 2415 (better than all but three of our 22 cases). The differences between the statics for the 2415 case and the disrupting statics are plotted in Fig. 24. There are no cycle skips in the figure! While the differences are smaller than in Fig. 23 , the differences are significant and range from -4.0 to 8.0 (the differences are not due to step size or round off).

When we saw the large differences between our best solutions and the "true solution" (the disrupting statics), we wondered about the source of the error. However, since the "true solution" does not maximize the stack power, we do not know the true solution for the stack power maximization problem for this data set. We conjecture that even the stack with the highest power possible for this data set may represent an unsatisfying solution showing patterns that look much like the "cycle skips" that we see in low power local solutions. We conclude that we cannot eliminate the cycle skips by maximizing the stack power. Our conclusion indicates that the problem is not as well posed as we would like. 


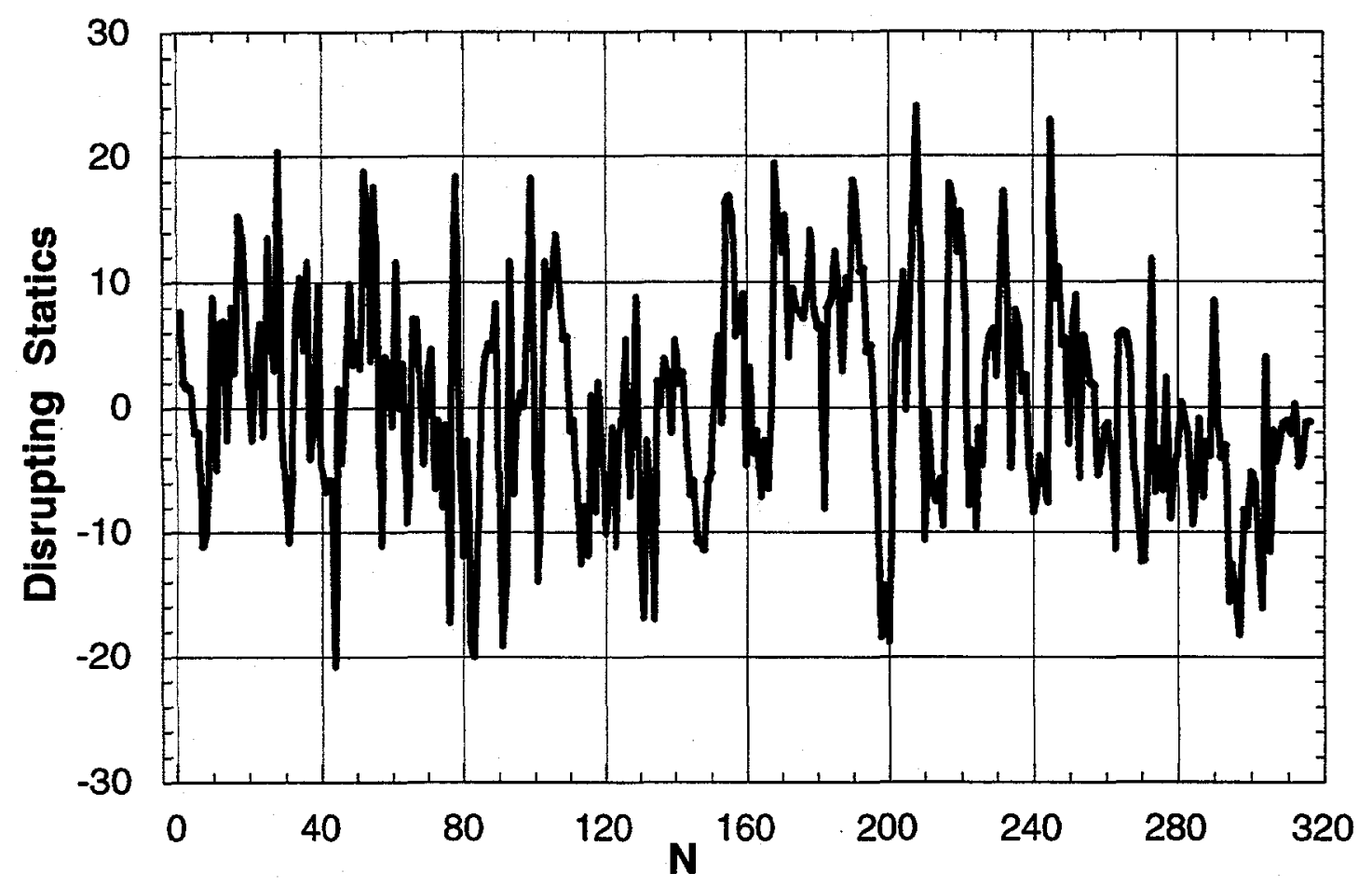

Fig. 20. The disrupting statics that were applied to the original seismic data (Fig. 5) to produce the input data for this project (Fig. 6).

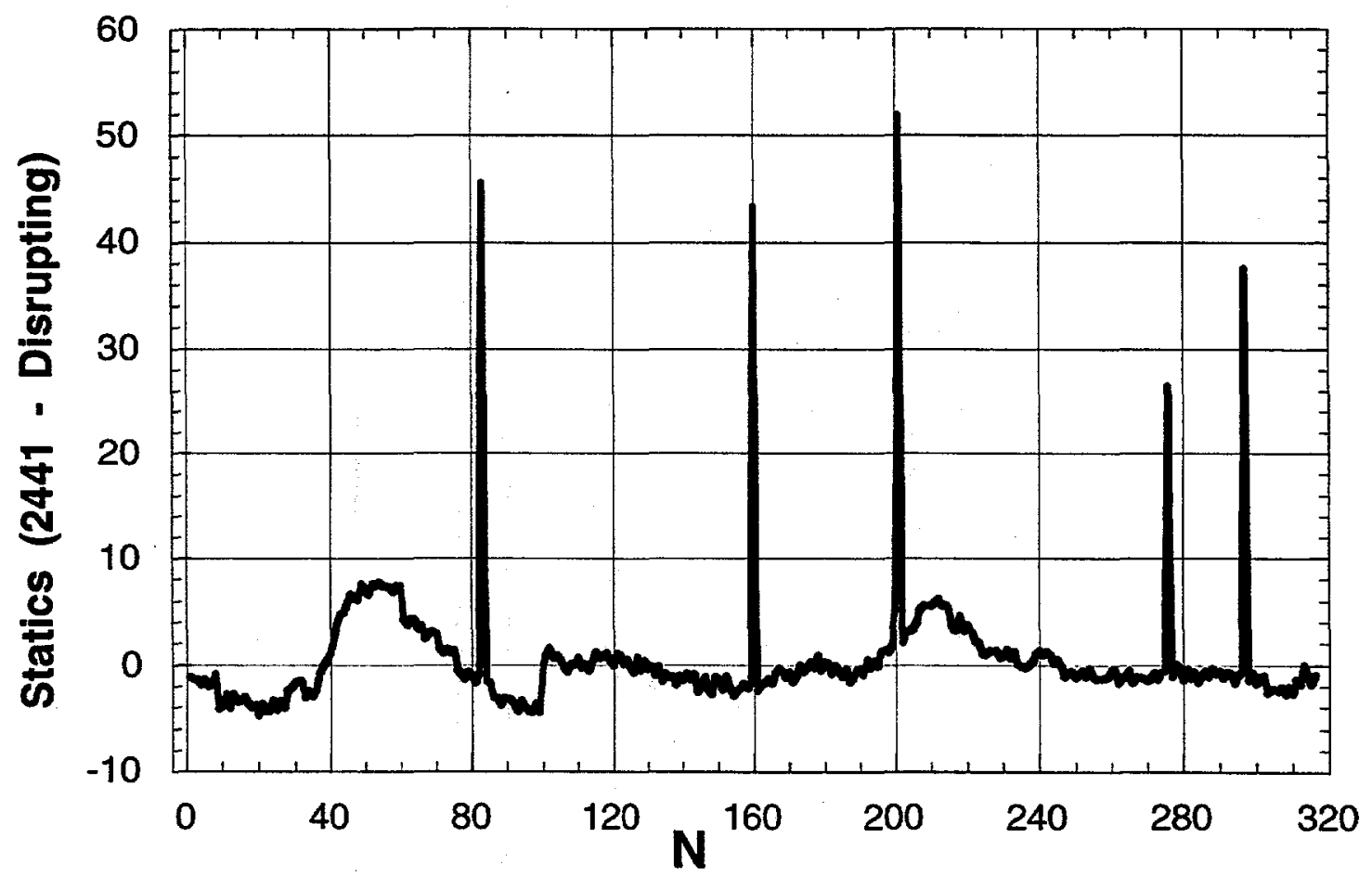

Fig. 21. The difference between the statics for the 2441 case and the disrupting statics. 


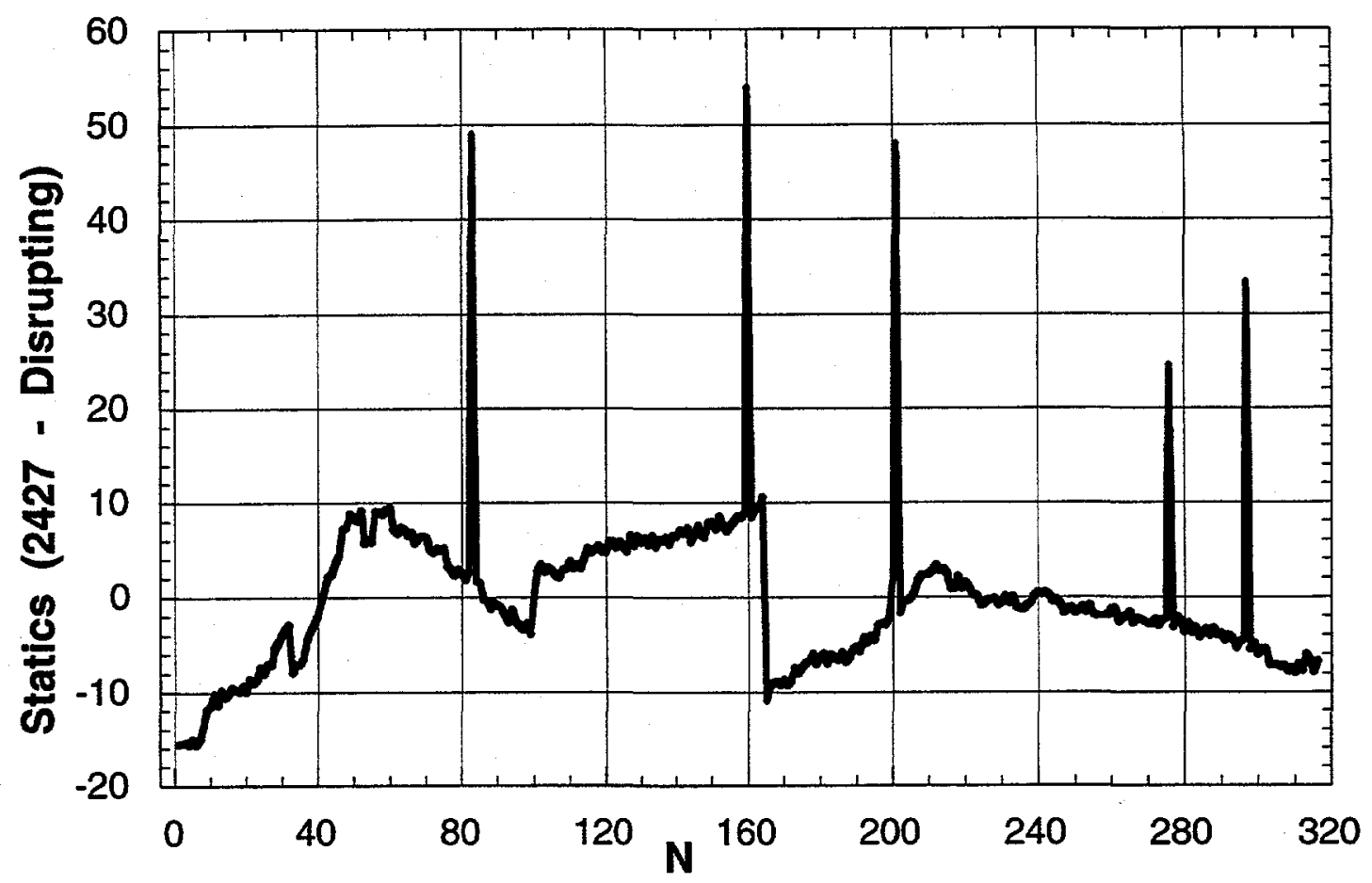

Fig. 22. The difference between the statics for the 2427 case and the disrupting statics.

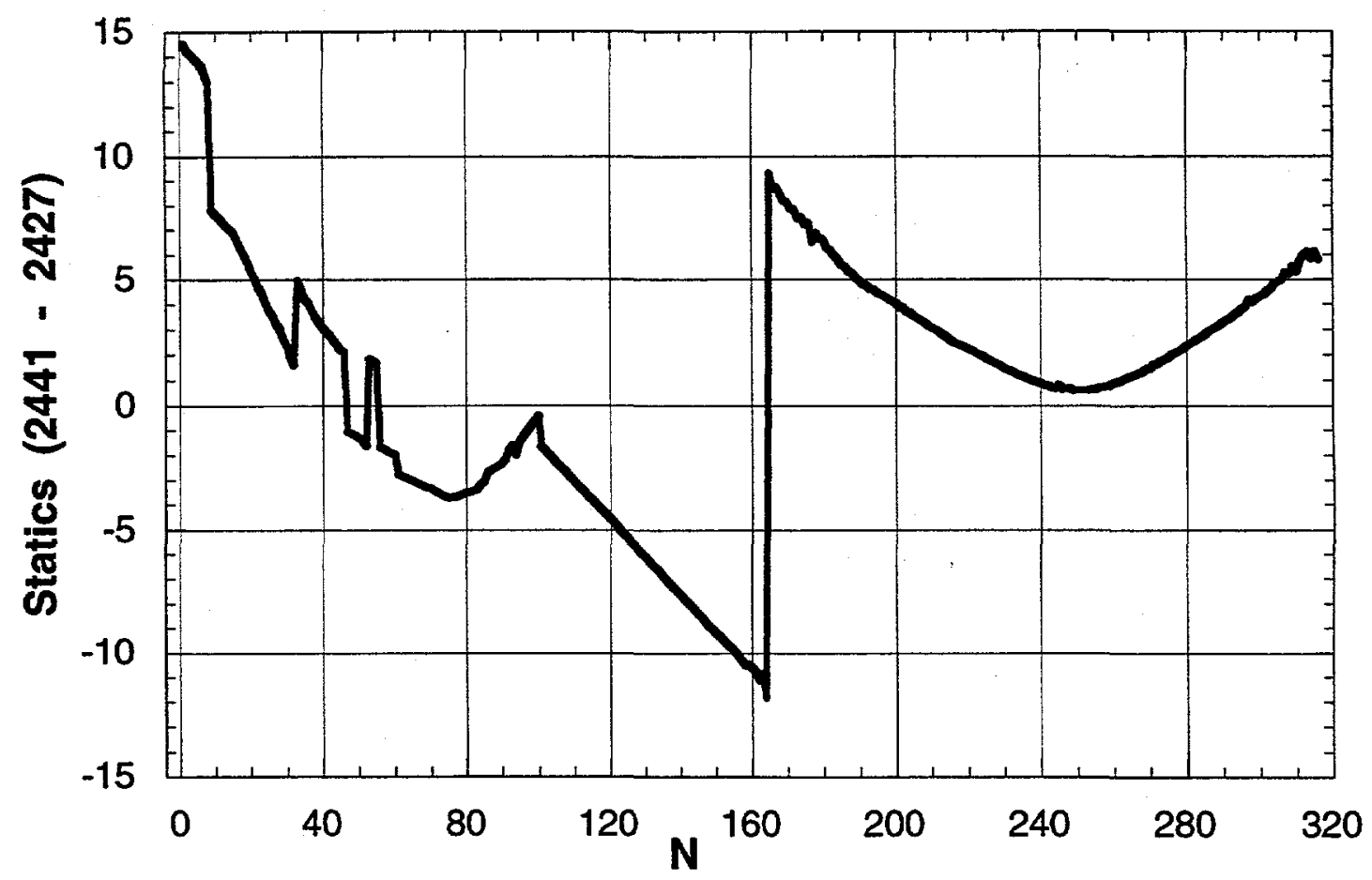

Fig. 23. The difference between the statics for the 2441 case and the 2427 case. 


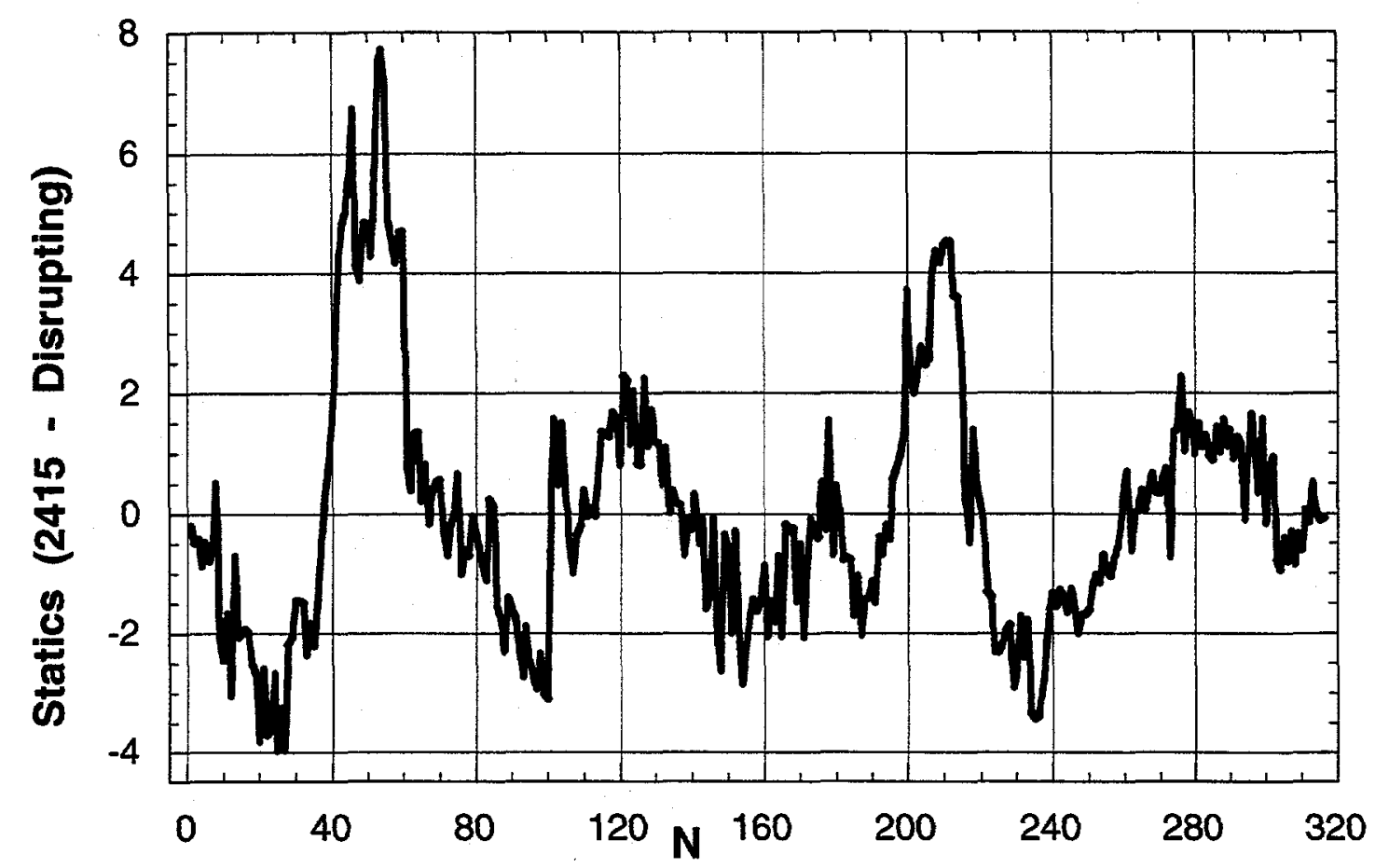

Fig. 24. The difference between the statics for the 2415 case and the disrupting statics.

\section{CONCLUSIONS}

Working in the frequency domain, we have used stack power maximization to estimate surface-consistent residual statics. Since the stack power objective function can have many local maxima, a global optimization method is required to find the optimum values for the residual statics. We had developed a new method (TRUST) for solving global optimization problems and had demonstrated it was superior to all competing methods for conventional benchmark problems. The residual statics problem can be very large with hundreds or thousands of parameters. In this paper, we have applied TRUST to the residual statics problem.

To solve this very challenging problem, we have made major improvements to TRUST and we have made several significant advances in the mathematical description of the residual statics problem. In our initial approach to the statics problem, we were using a deterministic version of TRUST that found the global minimum by executing a series of cycles of tunneling and descent. While the deterministic version of TRUST has been very successful in solving standard non-convex benchmark problems that have up to 20 parameters, it does not always find optimum values for large residual statics problems with 100 or more parameters. The basic reason that TRUST may be less successful with large problems is that the problem size increases exponentially with the number of parameters. 
In general, we cannot "solve" a global optimization problem. We must apply resource allocation: Apply finite resources (function evaluations) in an optimal way to find the best estimate of the global optimum. Since the deterministic version of TRUST does not always find optimum values for large residual statics problems, we enhanced TRUST by using one-dimensional tunneling paths. From a local minimum, we use the 1D version of TRUST to explore each of the $\mathrm{N}$ dimensions of the problem one at a time. When we find a point where the objective function has a lower value than the previous local minimum, we descend to the next local minimum. Using the 1D tunneling paths allowed us to find much better values for the stack power. Subsequently, Oblow developed SPT (Stochastic Pijavskij Tunneling) that greatly speeds up the 1D tunneling phase and has found many large values for the stack power.

The key parameter in SPT is the pseudo-Lipschitz constant which determines the slope of the two sides of the Pijavskij cone. The Pijavskij cones exclude regions of the search space. The SPT method has two other parameters (an estimate of the global minimum and the resolution) that can be used to estimate the pseudo-Lipschitz constant. We find that the Pijavskij cones quickly eliminate the search space and we usually evaluate the function about ten times to achieve a resolution of 100,000 points in the parameter range.

We have made several significant advances in the mathematical description of the residual statics problem. We have derived an upper bound for the stack power and defined a decoupled stack power. The solution of the decoupled problem both provides an initial estimate of the parameters for the coupled problem and provides a closer upper bound for the stack power of the coupled problem.

We have found many parameter vectors that give high values for the stack power. We have developed two methods for measuring the difference between two vectors: the Euclidean norm and the power norm. We remove the null space components before we calculate the Euclidean norm. We expect tradeoffs; as a parameter changes, the power will increase for some CMP and will decrease for others. The power norm is the sum of the absolute value of the differences in stack power for each CMP. The power norm is the best method for measuring the difference between two parameter vectors.

We have found many distinct parameter vectors that give high values for the stack power for a realistic sample problem that had been artificially created by large static disruptions. Since we knew the disrupting statics, we thought we knew the "true solution" for the sample problem. However, since the "true solution" does not maximize the stack power, we do not know the true solution for the stack power maximization problem for this data set. In this paper, we have argued that the exponential growth in difficulty prevents us from ever knowing "the solution" for a large global optimization problem. For this problem, the disaggregated problems provide a close upper bound for stack power. Since our best estimate (2441) is near the upper bound (2706) and the best of a large family of vectors with high stack power, we know we have found a very good estimate for the global maximum. 
An unexpected result was that high values of the stack power may not eliminate cycle skips.

\section{REFERENCES}

Barhen, J. and V. Protopopescu, 1996. "Generalized TRUST Algorithms for Global Optimization," in eds. C. A. Floudas, and P. M. Pardolas, State of the Art in Global Optimization: Computational Methods and Applications, Kluwer Academic Press, 163180.

Barhen, J., V. Protopopescu, and D. B. Reister, 1997. "TRUST: A Deterministic Algorithm for Global Optimization," Science, 276, 1094-1097.

Caklovic, K., and L. Caklovic, 1985. "Computation of Residual Statics Using Projectors," Geophysics, 50, 1502-1504.

Cambois, G., and P. L. Stoffa, 1992. "Surface-Consistent Deconvolution in the Log/Fourier Domain," Geophysics, 57, 823-840.

Cambois, G., and P. L. Stoffa, 1993. "Surface-Consistent Phase Decomposition in the Log/Fourier Domain," Geophysics, 58, 1099-1111.

Cetin, B. C., J. Barhen, and J. W. Burdick, 1993. "Terminal Repeller Unconstrained Subenergy Tunneling (TRUST) for Fast Global Optimization," Journal of Optimization Theory and Applications, 77, 97-126.

DuBose, J. B., Jr., 1993. "Practical Steps Toward Realizing the Potential of Monte Carlo Automatic Statics," Geophysics, 58, 399-407.

Levin, S. A., 1989. “Surface-Consistent Deconvolution," Geophysics, 54, 1123-1133.

Levy, S. and D. W. Oldenburg, 1987. "Automatic Phase Correction of CommonMidpoint Stacked Data," Geophysics, 52, 51-59.

Marcoux, M. O., 1981. "On The Resolution of Statics, Structure, and Residual Normal Moveout," Geophysics, 46, 984-993.

Marsden, D., 1993. "Static Corrections - A Review," The Leading Edge, 12, Part 1, 4349, Part 2, 115-120, Part 3, 210-216.

Morley, L., and J. Claerbout, 1983. "Predictive Deconvolution in Shot-Receiver Space," Geophysics, 48, 515-531. 
Normark, E., 1991. "Cooling Schedules for Simulated Annealing in the Residual Static Estimation Problem. Construction of Master Schedules," 53rd Mtg. Eur. Assoc. Expl Geophys., Abstracts, 91, 38-39.

Normark, E., 1993. "Residual Statics Estimation by Stack-Power Maximization in the Frequency Domain," Geophysical Prospecting, 41, 551-563.

Oblow, E. M. 1999. SPT: A Stochastic Tunneling Algorithm for Global Optimization, ORNL/TM-1339, Oak Ridge National Laboratory, Oak Ridge, TN.

Ronen, J. and J. F. Claerbout, 1985. "Surface-Consistent Residual Statics Estimation by Stack-Power Maximization," Geophysics, 50, 2759-2767.

Rothman, D. H., 1985. "Nonlinear Inversion, Statistical Mechanics, and Residual Statics Corrections," Geophysics, 50, 2784-2796.

Rothman, D. H., 1986. "Automatic Estimation of Large Residual Statics Corrections," Geophysics, 51, 332-346.

Sheriff, R. E. and L. P. Geldart, 1995. Exploration Seismology, Second Edition, Cambridge University Press.

Taner, M. T., F. Koehler, and K. A. Alhilali, 1974. "Estimation and Correction of NearSurface Time Anomalies," Geophysics, 39, 441-463.

Taner, M. T., and F. Koehler, 1981. "Surface Consistent Corrections," Geophysics, 46, 17-22.

Wiggins, R. A., K. L. Larner, and R. D. Wisecup, 1976. "Residual Statics Analysis as a General Linear Inverse Problem," Geophysics, 41, 922-938.

Wilson, W. G., W. G. Laidlaw, and K. Vasudevan, 1994. "Residual Statics Estimation Using the Genetic Algorithm," Geophysics, 59, 766-774.

Yilmaz, O., 1987. Seismic Data Processing, Society of Exploration Geophysics. 
ORNL/TN-1999/102

\section{INTERNAL DISTRIBUTION}

1. J. Barhen

9. N. S. V. Rao

2. B. J. Carr

3. W. E. Doll

10 - 35. D. B. Reister

4. R. R. Judkins

36. T. Zacharia

37. Central Research Library

5. E. M. Oblow

38. Document Reference Section

6. C. E. Oliver

39. Laboratory Records - RC

7. L. E. Parker

40. ORNL Patent Office

8. T. L. Payne

\section{EXTERNAL DISTRIBUTION}

43-47. J. B. DuBose, Jr., Paradigm Geophysical, 200 Smith Street, Suite 2100, Houston, TX 77002

48. R. E. Lemmon, FE-36, U.S. Department of Energy, 1 West 3rd Street, Williams Center Tower 1, Suite 1400, Tulsa, OK 74103

49. Dr. W. M. Polansky, SC-32, U.S. Department of Energy, 19901 Germantown Road, Germantown, MD 20874-1290

50. Dr. R. E. Price, SC-15, U.S. Department of Energy, 19901 Germantown Road, Germantown, MD 20874-1290

51. Dr. N. B. Woodward, SC-15, U.S. Department of Energy, 19901 Germantown Road, Germantown, MD 20874-1290 